\title{
Analytical Modeling of Multi-hop IEEE 802.15.4 Networks
}

\author{
Piergiuseppe Di Marco, Student Member, IEEE, Pangun Park, Member, IEEE, Carlo Fischione, Member, IEEE, \\ and Karl Henrik Johansson, Senior Member, IEEE
}

\begin{abstract}
Many of existing analytical studies of the IEEE 802.15.4 medium access control (MAC) protocol are not adequate because they are often based on assumptions such as homogeneous traffic and ideal carrier sensing, which are far from reality for multi-hop networks, particularly in the presence of mobility. In this paper, a new generalized analysis of the unslotted IEEE 802.15.4 MAC is presented. The analysis considers the effects induced by heterogeneous traffic due to multi-hop routing and different traffic generation patterns among the nodes of the network and the hidden terminals due to reduced carrier-sensing capabilities. The complex relation between MAC and routing protocols is modeled, and novel results on this interaction are derived. For various network configurations, conditions under which routing decisions based on packet loss probability or delay lead to an unbalanced distribution of the traffic load across multi-hop paths are studied. It is shown that these routing decisions tend to direct traffic toward nodes with high packet generation rates, with potential catastrophic effects for the node's energy consumption. It is concluded that heterogeneous traffic and limited carrier-sensing range play an essential role on the performance and that routing should account for the presence of dominant nodes to balance the traffic distribution across the network.
\end{abstract}

Index Terms-Hidden terminals, IEEE 802.15.4, medium access control (MAC), multi-hop.

\section{INTRODUCTION}

I EEE 802.15.4 is becoming the reference communication standard for wireless low-power and low-data-rate networks [1] with a wide range of applications in urban mobility, health care, industrial and environmental monitoring, building automation, smart grids, etc. Routing information over multi-hop paths is an important networking service for many of these applications [2]. To understand the fundamental performance limitations of IEEE 802.15.4 networks and suggest appropriate routing strategies, an accurate analytical model of the medium access control (MAC) for multi-hop topologies is instrumental.

Manuscript received July 7, 2011; revised December 18, 2011 and April 16, 2012; accepted April 25, 2012. Date of publication May 30, 2012; date of current version September 11, 2012. This work was supported in part by the Swedish Foundation for Strategic Research; by the Swedish Research Council; by the Swedish Governmental Agency for Innovation Systems; and by the European Union Project FeedNetBack, Project Hycon2, and Project Hydrobionets. The review of this paper was coordinated by Dr. H. Jiang.

P. Di Marco, C. Fischione, and K. H. Johansson are with the School of Electrical Engineering, Royal Institute of Technology, 10044 Stockholm, Sweden (e-mail: pidm@kth.se; carlofi@kth.se; kallej@kth.se).

P. Park was with the School of Electrical Engineering, Royal Institute of Technology, 10044 Stockholm, Sweden. He is now with the University of California, Berkeley, CA 94720 USA (e-mail: pgpark@kth.se).

Color versions of one or more of the figures in this paper are available online at http://ieeexplore.ieee.org.

Digital Object Identifier 10.1109/TVT.2012.2201221
While the performance of single-hop IEEE 802.15.4 star networks has been thoroughly investigated, there is not yet a clear understanding of the performance over multi-hop networks. For single-hop scenarios, many papers in the literature have proposed models for capturing the behavior of the IEEE 802.15.4 MAC, with saturated or unsaturated traffic, acknowledgements, and retransmissions [3]-[8]. The model presented in [5] is also validated through real test-bed experiments in [9]. These studies are based on extensions of the Markov chain model originally proposed by Bianchi for the IEEE 802.11 MAC protocol [10]. Based on these analytical models, the performance of the protocol can be improved by opportunely tuning the MAC parameters [9] or the duty cycle of the nodes [11]. In [12], the basic assumptions of the aforementioned Markov models are discussed, and the range of application is determined.

However, in all the proposed contributions, traffic is assumed to be homogeneous from node to node, in both saturated and unsaturated scenarios. This assumption is a major limitation in at least three important situations.

1) In single-hop networks, nodes may have different traffic generation rates as a result of the different services they provide, such as control applications with varying sampling rates.

2) In multi-hop networks, the traffic load varies according to the routing along the paths. Some nodes may experience heavier cross traffic, thus transmitting more packets than nodes that are traversed by fewer routing paths. It follows that the traffic is not homogeneous, regardless that the nodes generate their own packets at the same rate.

3) In networks with hidden terminals, the traffic sensed by the nodes is different from node to node, even when every node generates the same traffic. This is because some nodes may not perceive the ongoing transmissions of other nodes.

In the situations previously mentioned, which we believe are the most interesting and common, existing analytical studies of the IEEE 802.15.4 MAC are not adequate. In this paper, we attempt to overcome these limitations and propose a comprehensive analysis.

The remainder of this paper is organized as follows: In Section II, the related work and the original contribution are summarized. In Section III, we introduce the system model. In Section IV, we derive an analytical model of IEEE 802.15.4 MAC for multi-hop networks with heterogeneous traffic and hidden terminals. The accuracy of the model is evaluated by 
Monte Carlo simulations in Section V. Section VI concludes this paper and prospects our future work.

\section{RELATED WORKS AND ORIGINAL CONTRIBUtion}

The single-hop homogeneous models in [3]-[8] have been recently extended to cover some of the aspects of multi-hop heterogeneous networks. However, a comprehensive approach is still missing. Studies for single-hop IEEE 802.11 and IEEE 802.15.4 with heterogeneous traffic can be found in [13]-[15], where traffic classes are considered, but no hidden terminals. The effects of hidden terminals in homogeneous single-hop networks have been studied in [16] and [17]. In [18], multi-hop communication is modeled for IEEE 802.11 networks under single traffic flow. In [19], the work in [18] has been extended to multiple nonsaturated flows. In [20], a model for saturated traffic flows in IEEE 802.11 networks is presented. However, we note that these models cannot be directly applied to IEEE 802.15.4 networks due to the different access mechanisms of IEEE 802.11 MAC. In [21], a Markov chain model is presented for multi-hop IEEE 802.15.4 networks, but the model is limited to nodes that communicate to the coordinator through an intermediate relay node, which is assumed as not generating traffic and not competing for channel access. Therefore, to the best of our knowledge, there is still no analytical study in the literature that investigates the effect of routing over multi-hop networks using IEEE 802.15.4 MAC.

In this paper, we propose a novel analytical study that considers jointly routing and MAC, and we highlight the interdependence between routing decisions and end-to-end performance indicators. We provide an accurate model for small-scale networks and an approximate model that yields effective analysis of the performance for large-scale networks. We show how the IEEE 802.15.4 MAC may influence remarkably the routing alternatives. We study different performance indicators of IEEE 802.15.4 MAC over multi-hop networks, i.e., end-to-end reliability, end-to-end delay, and energy consumption. Specifically, the effect of the carrier-sensing range of nodes in different routing paths depends on the traffic. Thus, a different distribution of traffic load in the network determines different performances in terms of reliability, delay, and energy consumption of the routing. Based on the analysis proposed in this paper, we study conditions in which routing performance becomes critical for the load distribution and stability of the network.

Although there exists many routing protocol proposals in the literature, there is not yet a definite solution. Lively research activities and standardization efforts are ongoing, including the Routing Over Low power and Lossy networks (ROLL) working group of the Internet Engineering Task Force (IETF) [2], which is defining a routing protocol on top of the physical layer and MAC of IEEE 802.15.4. Therefore, we believe that our study may have an impact on the ongoing standardization.

\section{SYSTEM MODEL}

Consider a network of $N$ nodes, $V_{0}, \ldots, V_{N}$ that use the unslotted IEEE 802.15.4 MAC. We focus on this MAC modal-

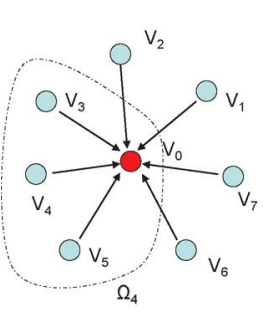

(a)

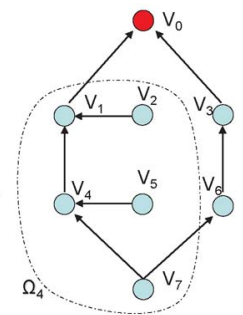

(b)

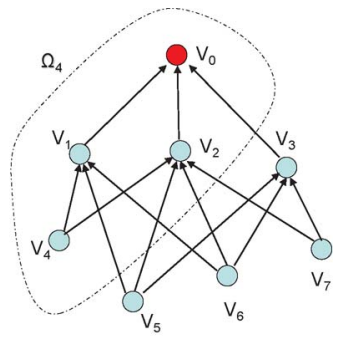

(c)
Fig. 1. Examples of (a) a single-hop topology, (b) a multi-hop topology with single end device, and (c) a multi-hop topology with multiple end devices for IEEE 802.15.4 networks. The dash-dotted area $\Omega_{4}$ delimits the carrier-sensing range of node $V_{4}$, i.e., the largest set of nodes that can be heard by $V_{4}$ while doing the CCA. The shape of $\Omega_{4}$ is irregular, because the carrier-sensing range may not always be isotropic. Our analysis incorporates any shape.

ity because it is common and relevant for the ROLL routing standardization [2]..$^{1}$ In the following, we illustrate the system model by considering three topologies, as shown in Fig. 1. However, the analytical results that we derive in this paper are general and not limited to a specific topology.

The topology in Fig. 1(a) refers to a single-hop (star) network where nodes forward their packets ${ }^{2}$ with single-hop communication to the root node $V_{0}$. In star networks, we denote by $l$ the link between $V_{l}$ and $V_{0}, l=1, \ldots, N$. The topologies in Fig. 1(b) and (c) are examples of multi-hop networks in which nodes forward traffic according to the uplink routing policy to $V_{0}$. In multi-hop networks, we label by $l, l=1, \ldots, G$, the link between a pair of communicating nodes $V_{i}$ and $V_{j}$, where $G$ is the number of such pairs.

For every node $V_{i}$, we define a neighborhood set $\Omega_{i}$, which contains all the nodes in the carrier-sensing range of $V_{i}$ (delimited by dash-dotted lines in the examples in Fig. 1). The carrier-sensing range is the set of nodes that can be heard by a node while performing the IEEE 802.15.4 clear channel assessment (CCA), which we describe later on. We denote by $\left|\Omega_{i}\right|$ the cardinality of $\Omega_{i}$. Note that the carrier-sensing radius is not necessarily isotropic. We assume that the channel is symmetric, so that, if $V_{k} \in \Omega_{i}$, then $V_{i} \in \Omega_{k}$, which is natural when transmitting and receiving over similar frequencies. For each link $\left(V_{i}, V_{j}\right)$, we define $\Omega_{j \backslash i}=\Omega_{j}-\Omega_{i}$ as the hidden node set of $V_{i}$ with respect to $V_{j}$, i.e., all nodes that are in the carrier-sensing range of receiver $V_{j}$ but that do not belong to the carrier-sensing range of transmitter $V_{i}$.

As a reference routing protocol, we consider the specifications of IETF ROLL [2]. The root node generates a destinationoriented directed acyclic graph (DODAG). In a DODAG, all edges are oriented such that no cycles exist. Directional routes in the network are indicated by arrows in Fig. 1. We define a parent set $\Gamma_{i} \subset \Omega_{i}$, which contains all nodes that may be next-hop nodes of $V_{i}$, and a children set $\Delta_{i} \subset \Omega_{i}$, which contains all nodes that have $V_{i}$ as next-hop node. The knowledge of the topological sets $\Omega_{i}$ and $\Gamma_{i}$ is then specified by IETF ROLL.

\footnotetext{
${ }^{1}$ The model derivation for the slotted mechanism follows similar steps as those presented in this paper, without a significant increase in complexity.

${ }^{2}$ Throughout this paper, we refer to packets as the MAC protocol data units.
} 
TABLE I

MAIN SYMBols USED IN THE PAPER

\begin{tabular}{|c|l|}
\hline Symbol & Meaning \\
\hline \hline$\alpha_{l}$ & Busy channel probability of node $V_{l}$ \\
\hline$\tau_{l}$ & Channel access probability of node $V_{l}$ \\
\hline$\Gamma_{l}$ & Parent set of node $V_{l}$ \\
\hline$\Delta_{l}$ & Children set of node $V_{l}$ \\
\hline$\Omega_{l}$ & Neighborhood set of node $V_{l}$ \\
\hline$\Omega_{l \backslash j}$ & Hidden node set of node $V_{l}$ \\
\hline$b_{i, k, j}^{l}$ & Stationary probability in the Markov chain \\
\hline$m_{0}$ & Initial backoff exponent \\
\hline$m_{b}$ & Maximum backoff exponent \\
\hline$m$ & Maximum number of backoffs \\
\hline$n$ & Maximum number of retransmissions \\
\hline$q_{l}$ & Packet generation probability of $V_{l}$ in idle state \\
\hline$q_{\mathrm{cf}, l}$ & Packet generation probability after CCA failure \\
\hline$q_{\mathrm{cr}, l}$ & Packet generation probability after retry limit \\
\hline$q_{\mathrm{succ}, l}$ & Packet generation probability after transmission \\
\hline$D_{l}$ & Packet delay of link $l$ \\
\hline$E_{\mathrm{tot}, l}$ & Energy consumption of node $V_{l}$ \\
\hline$G$ & Number of links in the network \\
\hline$L$ & Packet size \\
\hline$L_{\mathrm{ack}}$ & ACK length \\
\hline$M$ & Routing matrix \\
\hline$N$ & Number of nodes in the network \\
\hline$P_{\mathrm{blk}, l}$ & Blocking probability for node $V_{l}$ \\
\hline$P_{\mathrm{coll}, l}$ & Collision probability of node $V_{l}$ \\
\hline$Q_{l}$ & Aggregate traffic of node $V_{l}$ \\
\hline$R_{l}$ & Reliability of link $l$ \\
\hline$S_{b}$ & Backoff unit time \\
\hline$T$ & Traffic distribution matrix \\
\hline
\end{tabular}

We consider two multi-hop topologies in Fig. 1(b) and (c) to illustrate our analysis. In Fig. 1(b), there is one end-device $V_{7}$ and two main paths to the destination. Node $V_{7}$ may decide to route its packets either through nodes $V_{4}$ or $V_{1}$, which forward traffic also from nodes $V_{2}$ and $V_{5}$, or through $V_{6}$ and $V_{3}$. Note that route $V_{6}-V_{3}$ is less loaded in terms of traffic forwarding. In addition, we study the more complex routing graph in Fig. 1(c), where multiple end devices $\left(V_{4}, V_{5}, V_{6}\right.$, and $V_{7}$ ) may decide to route their packets either through nodes $V_{1}$, $V_{2}$, or $V_{3}$ to destination $V_{0}$. Coherently with the IETF ROLL specifications, we assume homogeneous link quality between a node and each one of its selected parents, because typically the parent set includes only nodes that can be reached with a guaranteed link quality. The actual forwarding decision is then based on routing metrics such as maximum end-to-end reliability or minimum end-to-end delay, which depend on the link performance at the MAC layer. As we show in Section V, the interaction between the MAC layer and routing decisions varies substantially between the routing paths, according to the carrier-sensing ranges.

In the next section, we introduce the general model for multihop unslotted IEEE 802.15.4 MAC, and we derive the basic relations with the routing policy. A list of the main symbols used in this paper is reported in Table I.

\section{Multi-Hop UnSLOtTEd IEEE 802.15.4 Medium ACCESS Control}

In this section, a generalized model of a heterogeneous unslotted IEEE 802.15.4 network is proposed. The analysis aims at deriving the network performance indicators, i.e., the reliability as probability of successful packet reception, the delay for successfully received packets, and the average node energy consumption. We first analyze the single-hop case, and then, we generalize the model equations to the multi-hop case. The main contribution of such a model, with respect to the Markov chain model in [5] or [22], is the presence of heterogeneous traffic with different node packet generation rates, hidden terminals, and multi-hop routing.

\section{A. CSMA/CA Mechanism of Unslotted IEEE 802.15.4}

Consider a node trying to transmit. In the unslotted carriersense multiple access with collision avoidance (CSMA/CA) of IEEE 802.15.4, first, the MAC layer initializes three variables, i.e., the number of backoffs $N B$, the backoff exponent $B E$, and the retransmissions counter $R T$. The default initialization is $N B=0, B E=\operatorname{macMin} B E$, and $R T=0$.

Then, the MAC layer delays for a random number of complete backoff periods in the range $\left[0,2^{B E}-1\right]$ units aUnitBackoff Period. When the backoff period is zero, the node performs a CCA. If the CCA is idle, then the node begins the packet transmission. The node experiences a delay of aTurnaroundTime to turn around from listening to transmitting mode. If the CCA fails due to busy channel, the MAC layer increases the value of both $N B$ and $B E$ by one up to a maximum value macMaxCSMABackoffs and macMaxBE, respectively. Hence, the values of $N B$ and $B E$ depend on the number of CCA failures of a packet. Once $B E$ reaches $\operatorname{macMax} B E$, it remains at the value of $\operatorname{macMax} B E$ until it is reset. If $N B$ exceeds macMaxCSMABackof $f s$, we assume that the packet is discarded due to channel access failure. Otherwise, the CSMA/CA algorithm generates a random number of backoff periods and repeats the process. If the channel access is successful, the node starts transmitting packets and waits for ACK. The reception of the corresponding ACK is interpreted as successful packet transmission. If the node fails to receive ACK due to collision or ACK timeout, the variable $R T$ is increased by one up to macMaxFrameRetries. If $R T$ is less than macMaxFrameRetries, the MAC layer initializes $B E=\operatorname{macMin} B E$ and follows the CSMA/CA mechanism to reaccess the channel. Otherwise, the packet is discarded due to the retry limit.

In the rest of this paper, we denote the IEEE 802.15.4 MAC parameters by $W_{0}=2^{\operatorname{macMin} B E}, m_{0}=\operatorname{macMin} B E$, $m_{b}=\operatorname{macMaxBE}, m=\operatorname{macMaxCSMABackoffs}, n=$ macMaxFrameRetries, and $S_{b}=a U$ nitBackoff Period.

\section{B. Single-Hop Network Model}

In this section, we develop new results for single-hop networks, which we then extend for multi-hop networks in Section IV-F. In particular, we derive the probability $\tau_{l}$ that node $V_{l}$ attempts a CCA in a randomly chosen time unit, the probability $\alpha_{l}$ that CCA is busy at link $l$, and the probability $P_{\text {coll }, l}$ that a transmitted packet encounters a collision at link $l$.

Let $s_{l}(t), c_{l}(t)$, and $r_{l}(t)$ be the stochastic processes representing the backoff stage, the state of the backoff counter, and the state of retransmission counter, respectively, which node $V_{l}$ experiences at time $t$. By assuming independent probability that 


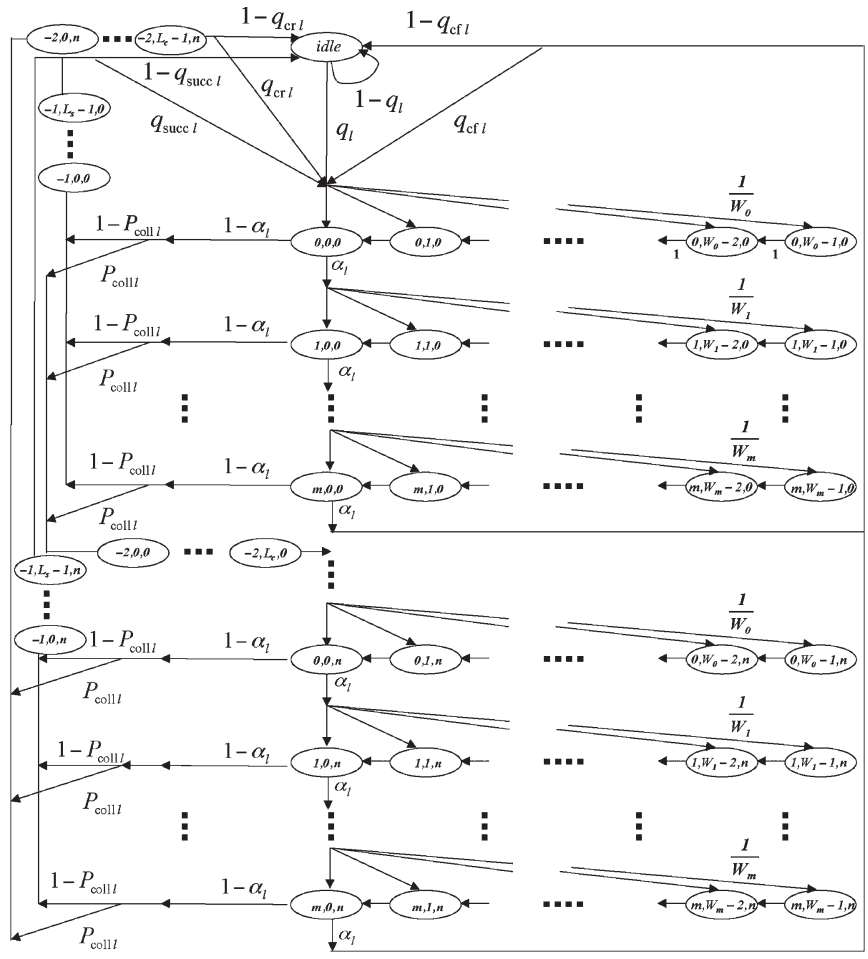

Fig. 2. Markov chain model of the CSMA/CA algorithm of a transmitting node of link $l$ for unslotted IEEE 802.15.4 MAC. Compared with existing works from the literature, this chain models the hidden terminal problem, heterogeneous traffic, and different packet generation rates per node.

nodes start sensing, the stationary probability $\tau_{l}$ that $V_{l}$ attempts carrier sensing in a randomly chosen slot time is constant. Then, the triple $\left(s_{l}(t), c_{l}(t), r_{l}(t)\right)$ is the 3 -D per-link Markov chain in Fig. 2, where we use $(i, k, j)$ to denote a particular state.

The Markov chain consists of four main parts corresponding to the idle state, backoff states, CCA states, and packet transmission states. The idle state corresponds to the idle-queue state when the node is waiting for the next packet generation time. The states from $\left(i, W_{m}-1, j\right)$ to $\left(i, W_{0}-1, j\right)$ represent the backoff states. The states $(i, 0, j)$ represent the CCA. The states $(-1, k, j)$ and $(-2, k, j)$ correspond to the successful transmission and packet collision, respectively.

The generation of unsaturated traffic at node $V_{l}$ is modeled by a packet generation probability in idle state $q_{l}$, i.e., the probability of generating a new packet in each time unit when the node is in idle state. Moreover, to include queueing effects of node buffers, we consider the probabilities of having a packet ready to be transmitted after the node has successfully sent a packet $q_{\text {succ, } l}$, after a packet has been discarded due to channel access failure $q_{\mathrm{cf}, l}$ or due to retry limit $q_{\mathrm{cr}, l}$. The expressions of the packet generation probabilities are derived in Appendix A.

We define the packet successful transmission time $L_{s}$ and the packet collision time $L_{c}$ as

$$
\begin{aligned}
& L_{s}=L+t_{\mathrm{ack}}+L_{\mathrm{ack}}+I F S \\
& L_{c}=L+t_{\mathrm{m}, \mathrm{ack}}
\end{aligned}
$$

where $t_{\text {ack }}$ is the ACK waiting time, IFS is the interframe spacing, and $t_{\mathrm{m} \text {,ack }}$ is the timeout of the ACK (see details in [1]). Packets are assumed to be all of the same length, consistently with the previous literature.

In the proposed Markov chain, $S_{b}$ is the unit time for all state transitions and corresponds to the transmission time of 20 symbols [1]. When performing CCA, a node is listening in RX mode for a duration of 8 symbols. Then, the nodes take a time of 12 symbols (aTurnaroundTime) to turn around from RX mode to TX mode before starting the transmission of the packet, which makes a total time of 20 symbols $\left(S_{b}\right)$ for a successful CCA. The length of packet $L$ and that of acknowledgement $L_{\text {ack }}$ are given as multiplies of $S_{b}$. Therefore, it is possible to conclude that $S_{b}$ is accurate enough to capture the main characteristics of the unslotted mechanism for a transmitting node.

By finding the stationary probabilities for each chain, we can derive the probability $\tau_{l}$ that node $V_{l}$ attempts CCA. Then, we couple all the per-link Markov chains to obtain a set of equations giving the network operating point, i.e., the busy channel probabilities $\alpha_{l}$ and the collision probabilities $P_{\text {coll, } l}$, for $l=1, \ldots, N$.

We define $b_{i, k, j}^{(l)}=\lim _{t \rightarrow \infty} \operatorname{Pr}\left[s_{l}(t)=i, c_{l}(t)=k, r_{l}(t)=\right.$ $j], i \in(-2, m), \quad k \in\left(0, \max \left(W_{i}-1, L_{s}-1, L_{c}-1\right)\right), j \in$ $(0, n)$, as the stationary distribution of the Markov chain of Fig. 2. We remark that these probabilities are associated to each link $l$. Then, we have the following result:

Proposition 4.1: Suppose that the probability to start sensing for every node is independent of the number of retransmissions suffered. Let $\alpha_{l}$ be the probability that CCA is busy, and let $P_{\text {coll }, l}$ be the probability that a transmitted packet encounters a collision, for $l=1, \ldots, N$. Then, the probability $\tau_{l}$ that a node $V_{l}$ attempts CCA in a randomly chosen time unit is

$$
\tau_{l}=\left(\frac{1-\alpha_{l}^{m+1}}{1-\alpha_{l}}\right)\left(\frac{1-y_{l}^{n+1}}{1-y_{l}}\right) b_{0,0,0}^{(l)}
$$

where (3), shown at the bottom of the next page, holds, and $y_{l}=P_{\text {coll }, l}\left(1-\alpha_{l}^{m+1}\right)$.

Proof: See Appendix B.

The probability $\tau_{l}$ given by the previous proposition depends on the probability $\alpha_{l}$ that CCA is busy and the probability $P_{\text {coll }, l}$ that a transmitted packet encounters a collision. We study these two probabilities next.

We derive the busy channel probability as follows:

$$
\alpha_{l}=\alpha_{\mathrm{pkt}, l}+\alpha_{\mathrm{ack}, l}
$$

where $\alpha_{\mathrm{pkt}, l}$ is the probability that node $V_{l}$ senses the channel and finds it occupied by a packet transmission in the neighborhood $\Omega_{l}$, whereas $\alpha_{\text {ack }, l}$ is the probability of finding the channel busy due to ACK transmission from $V_{0}$.

The probability that node $V_{l}$ finds the channel busy due to a packet transmission is the combination of two events: 1) At least one node accesses the channel in one of the previous $L$ time units, and 2) at least one of the nodes that accessed the channel found it clear. We would like to remark here a major difference with the Markov chain model proposed in [5] and [9]. In homogeneous networks with full sensing range, the busy channel probability is network information; it is the same for all 
the nodes. In heterogeneous networks, it depends on the access and busy channel probabilities of every node in the neighborhood. This introduces substantial analytical challenges.

Denote by $\mathcal{S}_{l}$ the event that node $V_{l}$ is sensing, and by $\mathcal{T}_{l}$ the event that node $V_{l}$ is transmitting. Denote also by $\mathcal{F}_{l}$ the event that there is at least one transmission in $\Omega_{l}$. Then

$$
\alpha_{\mathrm{pkt}, l}=\operatorname{Pr}\left[\mathcal{F}_{l} \mid \mathcal{S}_{l}\right]=\sum_{i=1}^{\left|\Omega_{l}\right|-1} \sum_{j=1}^{C_{l, i}} \operatorname{Pr}\left[\bigcup_{k=1}^{i} \mathcal{T}_{k_{j}} \mid \mathcal{S}_{l}\right]
$$

where

$$
C_{l, i}=\left(\begin{array}{c}
\left|\Omega_{l}\right|-1 \\
i
\end{array}\right)
$$

Index $k$ accounts for the events of simultaneous transmissions in the channel, and index $j$ enumerates the combinations of events in which a number $i$ of channel accesses are performed in the network simultaneously. Therefore, index $k_{j}$ refers to the node in the $k$ th position in the $j$ th combination of $i$ elements out of $\Omega_{l}$ so that

$\operatorname{Pr}\left[\bigcup_{k=1}^{i} \mathcal{T}_{k_{j}} \mid \mathcal{S}_{l}\right]=L \prod_{k=1}^{i} \tau_{k_{j}}\left(1-\prod_{k=1}^{i} \alpha_{k_{j}}\right) \prod_{h=i+1}^{\left|\Omega_{l}\right|}\left(1-\tau_{h_{j}}\right)$.

To illustrate (5), we consider Fig. 1(a) and assume that there are two contending nodes in the neighborhood of $V_{4}, \Omega_{4}=$ $\left\{V_{0}, V_{3}, V_{5}\right\}$. Note that $V_{0}$ does not generate packets. Then, the event of busy channel for node $V_{4}$, is given by the sum of three contributions.

1) Only node $V_{3}$ accessed the channel and found it clear. The probability of this event is $L \tau_{3}\left(1-\tau_{5}\right)\left(1-\alpha_{3}\right)$.

2) Only node $V_{5}$ accessed the channel and found it clear. Similarly to the previous case, the probability is $L \tau_{5}\left(1-\tau_{3}\right)\left(1-\alpha_{5}\right)$.

3) Both nodes accessed the channel and at least one node found it clear. Note that $V_{5}$ may not belong to $\Omega_{3}$ in this case. This probability is upper bounded by $L \tau_{3} \tau_{5}\left(1-\alpha_{3} \alpha_{5}\right)$.

Equation (5) follows as a generalization of this example.

The computation of the correlation among the busy channel probabilities is not an easy task. We use the upper bound $\left(1-\prod_{k=1}^{i} \alpha_{k_{j}}\right)$ because it represents a worst-case model scenario for the busy channel probability (uncorrelated busy channel events). As we show in Section V, this upper bound also provides a good approximation in the case of perfect sensing (maximum correlation). The reason is that this assumption affects only events in which two or more nodes are listening to the channel in the same time unit. However, in the case of homogeneous networks with full sensing range, the term $\left(1-\prod_{k=1}^{i} \alpha_{k_{j}}\right)$ can be replaced by the accurate expression $(1-\alpha)$.

A busy channel assessment due to ACKs depends on the probability of successful packet reception in $\Omega_{0}$. Let $R_{h}$ be the reliability of link $h$. It follows that

$$
\alpha_{\text {ack }, l}=L_{\text {ack }} \sum_{h \in \Omega_{0}, h \neq l} q_{h} R_{h}
$$

where $L_{\text {ack }}$ is the length of the ACK, and $q_{h}$ is the packet generation rate of node $V_{h}$. By summing up (5) and (6), we can compute $\alpha_{l}$ in (4).

We now turn our attention to the collision probability $P_{\text {coll }, l}$, i.e., the probability that the packet transmission from node $V_{l}$ to the root node $V_{0}$ encounters one or more simultaneous packet transmissions. Note that these transmissions may fully or partially overlap due to the limited size of the packets.

There are two main reasons of packet collisions for $V_{l}$.

1) Collision due to turnaround time: At least one node in $\Omega_{l}$ senses the channel idle while $V_{l}$ is in its turnaround after CCA, or at least one node in $\Omega_{l}$ is in its turnaround after CCA while $V_{l}$ senses the channel idle.

2) Collision due to hidden nodes: At least one node in $\Omega_{0 \backslash l}$ (hidden node) has started a packet transmission in one of the previous $L$ time units or before $V_{l}$ ends its transmission.

We define by $\mathcal{A}_{l}$ the event of collision due to turnaround time in $\Omega_{l}$ and by $\mathcal{B}_{l}$ the event of collision due to hidden nodes in $\Omega_{0 \backslash l}$. Therefore, the collision probability $P_{\text {coll }, l}$ is given by

$$
P_{\text {coll }, l}=\operatorname{Pr}\left[\mathcal{A}_{l}\right]+\operatorname{Pr}\left[\mathcal{B}_{l}\right]-\operatorname{Pr}\left[\mathcal{A}_{l}\right] \operatorname{Pr}\left[\mathcal{B}_{l}\right]
$$

Here, the probability of event $\mathcal{A}_{l}$ is given by the probability that at least one node in $\Omega_{l}$ accesses the channel and finds it free in

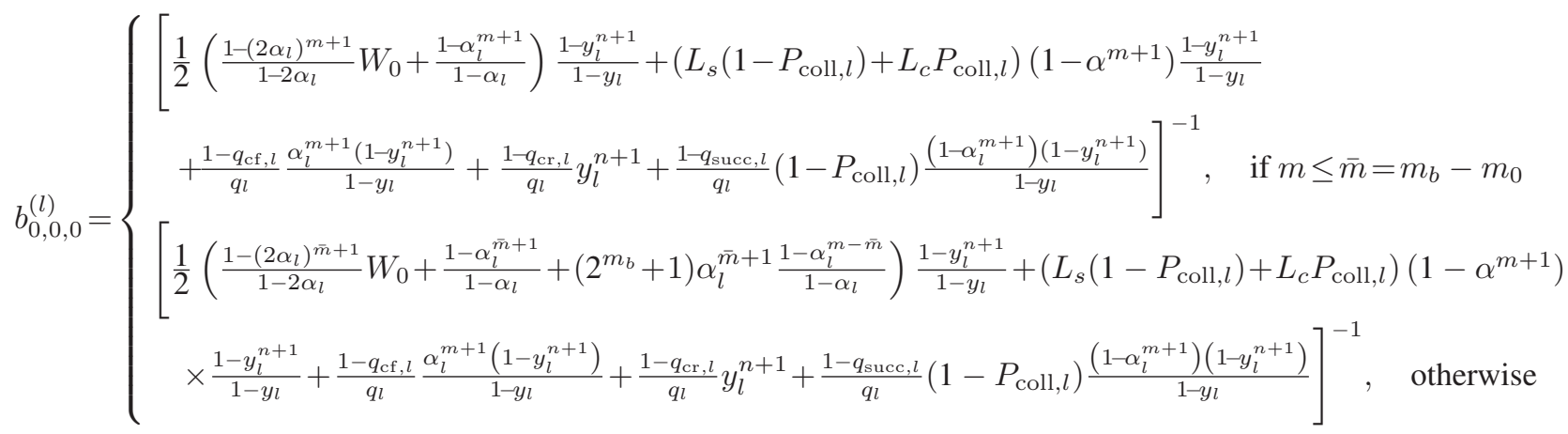


the same time unit (2aTurnaroundTime $\approx S_{b}$ ), i.e.,

$$
\operatorname{Pr}\left[\mathcal{A}_{l}\right]=\sum_{i=0}^{\left|\Omega_{l}\right|-1} \sum_{j=1}^{C_{l, i}} \prod_{k=1}^{i} \tau_{k_{j}}\left(1-\prod_{k=1}^{i} \alpha_{k_{j}}\right) \prod_{h=i+1}^{\left|\Omega_{l}\right|}\left(1-\tau_{h_{j}}\right) .
$$

Similarly, the probability of event $\mathcal{B}_{l}$ is given by

$\operatorname{Pr}\left[\mathcal{B}_{l}\right]=2 L \sum_{i=0}^{\left|\Omega_{0 \backslash l}\right|-1} \sum_{j=1}^{C_{l, i}} \prod_{k=1}^{i} \tau_{k_{j}}\left(1-\prod_{k=1}^{i} \alpha_{k_{j}}\right) \prod_{h=i+1}^{\left|\Omega_{0 \backslash l}\right|}\left(1-\tau_{h_{j}}\right)$.

In the following sections, we use these results to derive the expressions of the reliability, the delay for successfully received packets, and the energy consumption.

\section{Reliability}

In this section, we derive an expression of the reliability for each link of the network. We use the delivery ratio as a measure of the reliability. In the IEEE 802.15.4 CSMA/CA, packets are discarded due to either of the following reasons: 1) channel access failure or 2) retry limit. Channel access failure happens when a packet fails to obtain clear channel within $m+1$ backoffs. Furthermore, a packet is discarded if the transmission fails due to repeated collisions after $n+1$ attempts. Following the Markov model shown in Fig. 2, the probability that the packet is discarded due to channel access failure is

$$
\begin{aligned}
P_{\mathrm{cf}, l} & =\alpha_{l}^{m+1} \sum_{j=0}^{n}\left(P_{\mathrm{coll}, l}\left(1-\alpha_{l}^{m+1}\right)\right)^{j} \\
& =\frac{\alpha_{l}^{m+1}\left(1-\left(P_{\mathrm{coll}, l}\left(1-\alpha_{l}^{m+1}\right)\right)^{n+1}\right)}{1-P_{\mathrm{coll}, l}\left(1-\alpha_{l}^{m+1}\right)} .
\end{aligned}
$$

The probability of a packet discarded due to retry limit is

$$
P_{\mathrm{cr}, l}=\left(P_{\mathrm{coll}, l}\left(1-\alpha_{l}^{m+1}\right)\right)^{n+1} .
$$

Therefore, by using (8) and (9), the reliability is

$$
R_{l}=1-P_{\mathrm{cf}, l}-P_{\mathrm{cr}, l} .
$$

The expressions of the carrier-sensing probability $\tau_{l}$ in (2), the busy channel probability $\alpha_{l}$ in (4), and the reliability $R_{l}$ in (10), for $l=1, \ldots, N$, form a system of nonlinear equations that can be solved through numerical methods [23]. The solution of these equations provides us with the link reliability for the single-hop networks.

\section{Delay}

The total delay experienced in a successful packet transmission for node $V_{l}$ can be derived as $D_{l}=D_{l}^{s}+D_{l}^{q}$, where $D_{l}^{s}$ is the service time for a successfully received packet, and $D_{l}^{q}$ is the queueing delay.

The service time $D_{l}^{s}$ is defined as the time interval from the instant the packet is ready to be transmitted until an ACK for such a packet is received. If a packet is dropped due to either the limited number of backoffs $m$ or the finite retry limit $n$, its delay is not included into the derivation.

Let $D_{l, j}^{s}$ be the delay for a node that sends a packet successfully after $j$ unsuccessful attempts. From the Markov chain of Fig. 2, we derive the expected value of the delay $D_{l}^{s}$

$$
\mathbb{E}\left[D_{l}^{s}\right]=\sum_{j=0}^{n} \operatorname{Pr}\left[\mathcal{C}_{j} \mid \mathcal{C}\right] \mathbb{E}\left[D_{l, j}^{s}\right]
$$

where

$$
\mathbb{E}\left[D_{l, j}^{s}\right]=L_{s}+j L_{c}+\sum_{h=0}^{j} \mathbb{E}\left[T_{h}\right]
$$

$T_{h}$ is the backoff stage delay, and $L_{s}$ and $L_{c}$ are the time periods in the number of time units for successful packet transmission and collided packet transmission in (1). Event $\mathcal{C}_{j}$ denotes the occurrence of a successful packet transmission at attempt $j+1$, given $j$ previous unsuccessful transmissions, whereas event $\mathcal{C}$ denotes the occurrence of a successful packet transmission within $n$ attempts. We then have

$$
\begin{aligned}
\operatorname{Pr}\left[\mathcal{C}_{j} \mid \mathcal{C}\right] & =\frac{P_{\mathrm{coll}, l}^{j}\left(1-\alpha_{l}^{m+1}\right)^{j}}{\sum_{k=0}^{n}\left(P_{\mathrm{coll}, l}\left(1-\alpha_{l}^{m+1}\right)\right)^{k}} \\
& =\frac{\left(1-P_{\mathrm{coll}, l}\left(1-\alpha_{l}^{m+1}\right)\right) P_{\mathrm{coll}, l}^{j}\left(1-\alpha_{l}^{m+1}\right)^{j}}{1-\left(P_{\mathrm{coll}, l}\left(1-\alpha_{l}^{m+1}\right)\right)^{n+1}}
\end{aligned}
$$

where we recall that $P_{\text {coll }, l}$ is the collision probability, and $1-\alpha_{l}^{m+1}$ is the probability of successful channel access within the maximum number of $m$ backoff stages. Note that the probability of event $\mathcal{C}_{j}$ is normalized by considering all the possible events of successful attempts $\mathcal{C}$.

Let $T_{h, i}$ be the random time to obtain a successful CCA from the selected backoff counter value in backoff level $i$. By following a similar approach as that for the characterization of $D_{l}^{s}$, we see that the expected total backoff delay is modeled by $\mathbb{E}\left[T_{h}\right]=\sum_{i=0}^{m} \operatorname{Pr}\left[\mathcal{D}_{i} \mid \mathcal{D}\right] \mathbb{E}\left[T_{h, i}\right]$, where $T_{h, i}=(1+i)$ $T_{\mathrm{sc}}+\sum_{k=0}^{i} T_{h, k}^{b}, T_{\mathrm{sc}}$ is the sensing time in the unslotted mechanism, and $T_{h, k}^{b}$ is the backoff time at attempt $k$. Since $T_{h, k}^{b}$ is uniformly distributed in $\left[0, W_{k}-1\right]$, we can rewrite the expected backoff delay $\mathbb{E}\left[T_{h}\right]$ as

$$
\mathbb{E}\left[T_{h}\right]=T_{\mathrm{sc}}+\sum_{i=0}^{m} \operatorname{Pr}\left[\mathcal{D}_{i} \mid \mathcal{D}\right]\left(i T_{\mathrm{sc}}+\sum_{k=0}^{i} \frac{W_{k}-1}{2} S_{b}\right) .
$$

Event $\mathcal{D}_{i}$ denotes the occurrence of a busy channel for $i$ consecutive times and then that of an idle channel at the $i+1$ th time. The probability of $\mathcal{D}_{i}$ is conditioned to the successful sensing event within $m$ attempts $\mathcal{D}$, given that the node senses an idle channel in CCA. It follows that

$$
\operatorname{Pr}\left[\mathcal{D}_{i} \mid \mathcal{D}\right]=\frac{\alpha_{l}^{i}}{\sum_{k=0}^{m} \alpha_{l}^{k}}=\frac{\alpha_{l}^{i}\left(1-\alpha_{l}\right)}{1-\alpha_{l}^{m+1}}
$$


By replacing (12)-(15) in (11), the average service time for successfully received packets is obtained.

Following similar derivations, we compute the average service time for a packet that is discarded due to channel access failure $\mathbb{E}\left[D_{l}^{m}\right]$ as

$$
\mathbb{E}\left[D_{l}^{m}\right]=\sum_{j=0}^{n} \operatorname{Pr}\left[\mathcal{C}_{j} \mid \mathcal{C}\right] \mathbb{E}\left[D_{l, j}^{m}\right]
$$

where

$$
\mathbb{E}\left[D_{l, j}^{m}\right]=\sum_{h=0}^{j} \mathbb{E}\left[T_{h}\right]+(m+1) T_{\mathrm{sc}}+\sum_{k=0}^{m} \frac{W_{k}-1}{2} S_{b} .
$$

The average service time for a packet that is discarded due to the retry limit $\mathbb{E}\left\{D_{l}^{n}\right\}$ is given by

$$
\mathbb{E}\left[D_{l}^{n}\right]=L_{c}+\sum_{h=0}^{n} \mathbb{E}\left[T_{h}\right] .
$$

The expressions of the service time for successful transmission, channel access failure, and failure due to retry limit are used in Appendix A to compute the idle probabilities $q_{\mathrm{succ}, l}, q_{\mathrm{cf}, l}$, and $q_{\mathrm{cr}, l}$, respectively.

In the following, we focus on the queueing delay $D_{l}^{q}$ of node $V_{l}$. We consider a limited buffer size $B$ and describe the system as an $M / G / 1 / K$ queueing model [24]. Under these assumptions, the average queueing delay is

$$
\mathbb{E}\left[D_{l}^{q}\right]=\frac{1}{\lambda_{l}}\left(\sum_{n=1}^{B-1} n p_{l, k}+B\left(p_{l, 0}+\lambda_{l} \mathbb{E}\left[D_{l}^{s}\right]-1\right)\right)-\mathbb{E}\left[D_{l}^{s}\right]
$$

where probabilities $p_{l, k}$ are derived in Appendix A.

\section{E. Energy Consumption}

Here, we develop the expression of the energy consumption. By considering the Markov chain model in Fig. 2, the average energy consumption of node $V_{l}$ is given by

$$
\begin{aligned}
E_{\text {tot }, l}= & P_{i} \sum_{i=0}^{m} \sum_{k=1}^{W_{i}-1} \sum_{j=0}^{n} b_{i, k, j}^{(l)}+P_{\mathrm{sc}} \sum_{i=0}^{m} \sum_{j=0}^{n} b_{i, 0, j}^{(l)} \\
& +P_{t} \sum_{j=0}^{n} \sum_{k=0}^{L-1}\left(b_{-1, k, j}^{(l)}+b_{-2, k, j}^{(l)}\right) \\
& +P_{i} \sum_{j=0}^{n}\left(b_{-1, L, j}^{(l)}+b_{-2, L, j}^{(l)}\right) \\
& +\sum_{j=0}^{n} \sum_{k=L+1}^{L+L_{\text {ack }}+1}\left(P_{r} b_{-1, k, j}^{(l)}+P_{i} b_{-2, k, j}^{(l)}\right)+P_{\mathrm{sp}} b_{\text {idle }}^{(l)}
\end{aligned}
$$

where $P_{i}, P_{\mathrm{sc}}, P_{t}, P_{r}$, and $P_{\mathrm{sp}}$ are the average energy consumption in idle-listening, channel-sensing, transmitting, receiving, and idle-queueing states, respectively. We assume that the radio is set in idle-listening state during the backoff stages and the timeout of ACK $t_{\mathrm{m} \text {,ack }}=L_{\mathrm{ack}}+1$, in time units $S_{b}$. In (20), the first and second terms take into account the energy consumption during idle backoff state and channel-sensing state, respectively. The third, fourth, and fifth terms consider the energy consumption of the packet transmission stage. The last term is the energy consumption of the idle-queue stage without packet generation. By following the computation of the terms $b_{i, k, j}^{(l)}$ in (39) into (20), we obtain the average energy consumption in closed form. In the next section, we generalize the analysis to the multi-hop case.

\section{F. Extension to Multi-hop Networks}

In a multi-hop topology, the total number of links $G$ is not equal to the number of nodes $N$. Recall that we associate to every link $l$ a transmitter-receiver pair $\left(V_{i}, V_{j}\right)$. The proposed Markov chain model is extended to a generic network in which information is routed through multi-hop communications to a root node. The Markov chain model should be solved for each link of the network by considering now that the generic destination node $V_{j}$ in each link has a neighborhood $\Omega_{j}$ and forwards an aggregate traffic $Q_{j}$.

Let $\lambda=\left[0, \lambda_{1}, \ldots, \lambda_{N}\right]$ be a vector of node traffic generation rates, where each component is associated with a node. In addition to $\lambda_{i}$, node $V_{i}$ has to forward traffic generated by nodes in its children set $\Delta_{i}$. We measure the total average aggregated traffic in link $l$ as $Q_{l}=q_{l} / S_{b} \mathrm{pkt} / \mathrm{s}$, where $q_{l}$ is the probability of having a packet to transmit in each time unit (see Fig. 2) and $S_{b}$ is the duration of the basic time unit in IEEE 802.15.4.

The aim of the following analysis is to investigate the total traffic load $Q_{l}$, which we associate to probability $q_{l}$ in the perlink Markov chain. To do so, we must characterize the traffic distribution in the network according to a routing policy.

Define $\pi_{i, j}$ as the metric associated to link $\left(V_{i}, V_{j}\right)$ to build the routing graph, as specified by IETF ROLL. In a practical example, $\pi_{i, j}$ may be the end-to-end reliability or delay from node $V_{i}$ to $V_{0}$, by choosing node $V_{j}$ as the next-hop node. At the routing layer, metrics are chosen to be static if the network is stationary. However, due to the dynamic nature of wireless connectivity, link attributes including reliability and delay may change over time, and the routing metrics are updated accordingly. We can represent this dynamical behavior using a statistical analysis. The effect of routing can be described by a matrix $\mathbf{M} \in \mathbb{R}^{(\mathrm{N}+1) \times(\mathrm{N}+1)}$, in which element $M_{i, j}$ corresponds to the probability that the metric in link $l=\left(V_{i}, V_{j}\right)$ is the highest among the set of candidate receivers $\Gamma_{i}$, i.e.,

$$
M_{i, j}=\operatorname{Pr}\left[\pi_{i, j}=\max _{V_{h} \in \Gamma_{i}} \pi_{i, h}\right] .
$$

The distribution of the traffic flows along the network can be modeled by matrix $\mathbf{M}$ and by scaling it by the probability of successful reception in each link (only successfully received packets are forwarded). Therefore, we define a matrix T such that $T_{i, j}=M_{i, j} R_{l}$, where $R_{l}$ is the reliability in link $l=\left(V_{i}, V_{j}\right)$, as derived in Section IV-C. The aggregate traffic vector $Q=\left[0, Q_{1}, \ldots, Q_{N}\right]$ is the sum of generated traffic and forwarded traffic from children nodes, which gives the system 


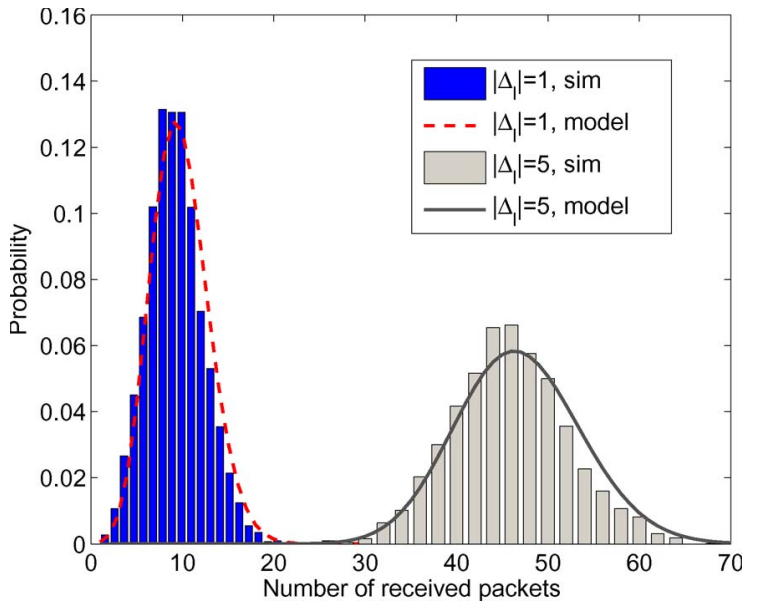

Fig. 3. PDF of the number of received packets in a period $T=10 \mathrm{~s}$ for a destination node $V_{l}$ by varying the number of children nodes in $\Delta_{l}$. The network is composed of $N=14$ nodes, with traffic rate $\lambda_{l}=5 \mathrm{pkt} / \mathrm{s}$, for $l=1, \ldots, N$.

of flow balance equations $Q=\lambda+Q \mathbf{T}$. In steady state, we have

$$
Q=\lambda[\mathbf{I}-\mathbf{T}]^{-1}
$$

where $\mathbf{I} \in \mathbb{R}^{(\mathrm{N}+1) \times(\mathrm{N}+1)}$ is the identity matrix. Since the routing graph is assumed to be acyclic, matrix $\mathbf{T}$ has spectral radius less than one. Therefore, it is easy to show that the inverse matrix $[\mathbf{I}-\mathbf{T}]^{-1}$ always exists.

Equation (21) gives the relation between the idle packet generation probability $q_{l}$, the effect of routing (through matrix $\mathbf{M}$ ), and the performance at the MAC layer (through link reliability $R_{l}$ ). To obtain the multi-hop network model, we couple (21) with the expressions for $\tau_{l}, \alpha_{l}$, and $R_{l}$, as obtained by (2), (4), and (10), respectively.

We recall that the packet generation follows a Poisson distribution. In the derivation of the total aggregate traffic $Q$, we assume that the forwarded traffic also follows a Poisson distribution. This assumption holds if the distribution of the number of received packets at node $V_{j}$ can be approximated by a Poisson distribution. To validate this approximation, in Fig. 3, we show the probability density function (pdf) of the number of received packets in a time interval of $10 \mathrm{~s}$ in a single-hop network with $N=14$ nodes and traffic generation rates $\lambda_{i}=5 \mathrm{pkt} / \mathrm{s}$ using Monte Carlo simulations. Details of the simulation environment are given in Section V. We observe that a Poisson distribution with arrival rate $Q_{j}-\lambda_{j}$ predicts well the pdf of the number of received data packets.

Another important consideration in the extension to multihop networks is the fact that ACKs might be sent from any node in the network, according to the distribution of the traffic. In the derivation of the busy channel probability due to ACK transmission $\alpha_{\text {ack }, l}^{\mathrm{mh}}$, we replace (6) with

$$
\alpha_{\mathrm{ack}, l}^{\mathrm{mh}}=L_{\mathrm{ack}} \sum_{j \in \Omega_{i}} \sum_{h \in \Delta_{j}} q_{h} T_{i, j}
$$

which includes the effect of limited carrier-sensing range at the destination and the routing matrix $\mathbf{M}$. Recall that $\Delta_{j}$ is the set of children nodes of $V_{j}$ and that index $i$ refers to the transmitting node in link $l$.

There are extra challenges in the derivation of the collision probability $P_{\text {coll }, l}$. In particular, a packet from node $V_{i}$ might collide with an ACK from a node in the hidden node set. In the simplest situation, this event involves four nodes. There is a collision due to a hidden node ACK if a packet from $V_{i}$ is transmitted to $V_{j}$ while $V_{k} \notin\left\{\Omega_{i}+\Omega_{j}\right\}$ successfully transmits a packet to $V_{h} \in \Omega_{j \backslash i}$. Then, the ACK from $V_{h}$ leads to a collision with the packet from $V_{i}$ at receiver $V_{j}$. The derivation of the probability of these events is not trivial and computationally demanding. In our model, we assume, for simplicity, that ACKs from hidden nodes do not prevent the destination node from successfully receiving a packet. This hypothesis is supported by the fact that the time in which ACKs and packets may overlap is short with respect to the packet transmission time (i.e., $L_{\text {ack }} \ll L$ ).

Moreover, packets might be dropped at the receiver due to the limited buffer size $B$. We include these events by considering the blocking probability for each node $P_{\mathrm{blk}, l}$, i.e., the packet is discarded at the receiver because the queue is full. For the $\mathrm{M} / \mathrm{G} / 1 / \mathrm{K}$ queueing model [24], we get

$$
P_{\mathrm{blk}, l}=1-\frac{1}{p_{l, 0}+Q_{l} \mathbb{E}\left\{D_{l}^{s}\right\}}
$$

where $\mathbb{E}\left\{D_{l}^{s}\right\}$ is the average delay in (11), and $p_{l, 0}$ is derived in Appendix A.

We derive the end-to-end reliability of $V_{i}$ by the product of each link reliability in the path to $V_{0}$. Similarly, the end-to-end delay is the sum of the delays in the path from transmitter $V_{i}$ to the root node $V_{0}$. The energy consumption for every node considers that nodes are in idle-listening state during the idlequeuing stage in the Markov chain and include the cost for receiving packets and transmitting ACKs. Consequently, (20) is extended in the multi-hop case to

$$
\begin{aligned}
E_{\mathrm{tot}, \mathrm{l}}^{\mathrm{mh}}= & P_{i} \sum_{i=0}^{m} \sum_{k=1}^{W_{i}-1} \sum_{j=0}^{n} b_{i, k, j}^{(l)}+P_{\mathrm{sc}} \sum_{i=0}^{m} \sum_{j=0}^{n} b_{i, 0, j}^{(l)} \\
& +P_{t} \sum_{j=0}^{n} \sum_{k=0}^{L-1}\left(b_{-1, k, j}^{(l)}+b_{-2, k, j}^{(l)}\right) \\
& +P_{i} \sum_{j=0}^{n}\left(b_{-1, L, j}^{(l)}+b_{-2, L, j}^{(l)}\right) \\
& +\sum_{j=0}^{n} \sum_{k=L+1}^{L+L_{\mathrm{ack}}+1}\left(P_{r} b_{-1, k, j}^{(l)}+P_{i} b_{-2, k, j}^{(l)}\right) \\
& +\left(P_{r} L+P_{i}+P_{t} L_{\mathrm{ack}}\right) \sum_{h \in \Delta_{i}} q_{h} T_{h, j} b_{\text {idle }}^{(l)} \\
& +P_{i}\left(1-\left(L+L_{\mathrm{ack}}+1\right) \sum_{h \in \Delta_{i}} q_{h} T_{h, j}\right) b_{\text {idle }}^{(l)} .
\end{aligned}
$$

We validate the analysis in Section V. 
TABLE II

CARRIER-SENSING RANGE IN THE NETWORK SCENARIOS IN FIG. 1

\begin{tabular}{|c|c|c|c|c|c|}
\hline & $\begin{array}{c}\text { Fig. 1a } \\
\text { reduced sens. }\end{array}$ & $\begin{array}{c}\text { Fig. 1b } \\
\text { non-interf. }\end{array}$ & $\begin{array}{c}\text { Fig. 1b } \\
\text { interfering }\end{array}$ & $\begin{array}{c}\text { Fig. 1c } \\
\text { non-interf. }\end{array}$ & $\begin{array}{c}\text { Fig. 1c } \\
\text { interfering }\end{array}$ \\
\hline$V_{1}$ & $V_{0}, V_{2}, V_{7}$ & $V_{0}, V_{2}, V_{3}, V_{4}, V_{5}$, & $\left\{V_{i}\right\}, i \neq 1,3$ & $\left\{V_{i}\right\}, i \neq 1,7$ & $\left\{V_{i}\right\}, i \neq 1$ \\
\hline$V_{2}$ & $V_{0}, V_{1}, V_{3}$ & $V_{0}, V_{1}, V_{4}, V_{5}$ & $\left\{V_{i}\right\}, i \neq 2$ & $\left\{V_{i}\right\}, i \neq 2$ & $\left\{V_{i}\right\}, i \neq 2$ \\
\hline$V_{3}$ & $V_{0}, V_{2}, V_{4}$ & $V_{0}, V_{1}, V_{6}$ & $\left\{V_{i}\right\}, i \neq 1,3$ & $\left\{V_{i}\right\}, i \neq 3,4$ & $\left\{V_{i}\right\}, i \neq 3$ \\
\hline$V_{4}$ & $V_{0}, V_{3}, V_{5}$ & $V_{1}, V_{2}, V_{5}, V_{7}$ & $\left\{V_{i}\right\}, i \neq 4$ & $V_{0}, V_{1}, V_{2}$ & $\left\{V_{i}\right\}, i \neq 4$ \\
\hline$V_{5}$ & $V_{0}, V_{4}, V_{6}$ & $V_{1}, V_{2}, V_{4}, V_{7}$ & $\left\{V_{i}\right\}, i \neq 5$ & $V_{0}, V_{1}, V_{2}, V_{3}$ & $\left\{V_{i}\right\}, i \neq 5$ \\
\hline$V_{6}$ & $V_{0}, V_{5}, V_{7}$ & $V_{3}, V_{7}$ & $\left\{V_{i}\right\}, i \neq 6$ & $V_{0}, V_{1}, V_{2}, V_{3}$ & $\left\{V_{i}\right\}, i \neq 6$ \\
\hline$V_{7}$ & $V_{0}, V_{1}, V_{6}$ & $V_{4}, V_{5}, V_{6}$ & $\left\{V_{i}\right\}, i \neq 0,7$ & $V_{0}, V_{2}, V_{3}$ & $\left\{V_{i}\right\}, i \neq 7$ \\
\hline
\end{tabular}

\section{G. Approximate Model for Large-Scale Networks}

In the previous sections, we derived closed-form expressions of the performance indicators in single-hop and multi-hop IEEE 802.15.4 networks. However, the computation time of the Markov chain parameters $\alpha_{\mathrm{pkt}, i}$ and $P_{\mathrm{coll}, i}$ in (5) and (7) exponentially increases with the number of neighboring nodes $\left|\Omega_{i}\right|$ and the number of hidden nodes $\left|\Omega_{j \backslash i}\right|$. Therefore, the computation of these parameters becomes unpractical when any of these numbers becomes greater than 15-20.

In this section, we propose approximate expressions of $\alpha_{i}$ and $P_{\text {coll }, i}$, which are linear with the number of neighboring and hidden nodes and can be used to derive the performance indicators for large-scale networks. In particular, we consider the event $\mathcal{U}_{i}$ that the channel is busy in $\Omega_{i}$ for a given time unit. The probability of this event can be approximated by multiplying the probability that at least one node in $\Omega_{i}$ accesses the channel and the average probability of idle channel during CCA in $\Omega_{i}$, i.e.,

$\operatorname{Pr}\left(\mathcal{U}_{i}\right)=\frac{1}{\left|\Omega_{i}\right|-1}\left(1-\prod_{l \in \Omega_{i}}\left(1-\tau_{l}\right)\right) \sum_{k \in \Omega_{i}}\left(1-\alpha_{k}\right)$.

The busy channel probability due to packet transmission $\alpha_{\text {pkt }, i}$ and the collision probability $P_{\mathrm{coll}, i}$ can be then written as

$$
\begin{aligned}
& \alpha_{\mathrm{pkt}, i}=L \operatorname{Pr}\left(\mathcal{U}_{i}\right) \\
& P_{\text {coll }, i}=\operatorname{Pr}\left(\mathcal{U}_{i}\right)+\left(1-\operatorname{Pr}\left(\mathcal{U}_{i}\right)\right) \operatorname{Pr}\left(\mathcal{U}_{j \backslash i}\right) .
\end{aligned}
$$

When the number of nodes in $\Omega_{i}$ is small, the previous equations may lead to inaccurate estimation of the parameters due to the averaging operation. However, this model becomes more accurate as the size of the set increases, because the variance of the distribution of busy channel probabilities reduces. The approximate model is validated through simulations in the next section.

\section{Simulation Results}

In this section, we present extensive Monte Carlo simulations to validate our analysis. The simulations are based on the specifications of the IEEE 802.15.4 [1] with several values of the traffic pattern and carrier-sensing range. In our implementation, time is slotted and synchronized with respect to a simulation clock $S_{b}=320 \mu$ s. However, in each iteration, we keep into account the asynchronous behavior of the nodes during CCA and packet transmission by dividing the slot into subslots of $16 \mu \mathrm{s}$, which corresponds to the symbol period in the standard.

According to the IETF ROLL specifications [2], typical applications of interest in urban, industrial, or home environments operate with packet generation rates of the order of few packets per minute. However, to validate our model in more critical situations, we also consider traffic rates on the order of packets per second. We set the MAC parameters to $m_{0}=3, m=4$, $m_{b}=7, n=[0,1], L=7$, and $L_{\text {ack }}=2$. Other settings give results similar to those discussed next. First, we consider singlehop and then multi-hop networks.

\section{A. Single-Hop Network}

In the first set of simulation results, we validate the model proposed in Section IV-B for a single-hop topology [see Fig. 1(a)].

To study the impact of hidden terminals, we consider two basic scenarios, i.e., $\left|\Omega_{l}\right|=N$, which is denoted by full-sensing capability, and $\left|\Omega_{l}\right|=3$, which represents reduced-sensing capability (the neighborhood is composed by the root node $V_{0}$ and two adjacent nodes). The list of nodes in the reduced carriersensing range of each node is reported in Table II.

Fig. 4 shows the average reliability computed over all the links for a single-hop network with homogeneous traffic. On the $x$-axis, the node packet generation rate is reported. Results are shown for different sizes of the network $(N=7, N=14)$ and by considering both full- and reduced-sensing capabilities. As a reference, we report the reliability obtained from the singlehop homogeneous model with full-sensing capabilities, which was presented in [5] and experimentally validated in [9], and here is adapted to the unslotted mechanism of IEEE 802.15.4. A good agreement between simulations and analytical results is observed. By reducing the carrier-sensing capabilities, the model proposed in this paper allows us to observe a negative impact on the reliability, which cannot be predicted by the earlier model of [5]. However, in the case of full-sensing capabilities, our proposed model coincides with the unslotted version proposed in [5]. There is a small gap between the two 


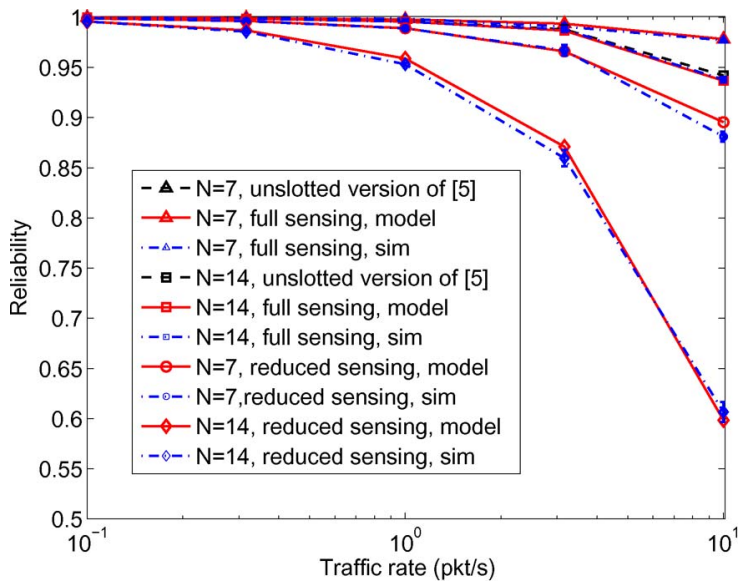

Fig. 4. Reliability versus traffic rate $\lambda$ for the single-hop topology in Fig. 1(a). Full sensing and reduced sensing correspond to $\left|\Omega_{l}\right|=N$ and $\left|\Omega_{l}\right|=3$, respectively. Unslotted version of [5] refers to the single-hop model presented in [5] and experimentally validated in [9], which is limited only to the homogeneous case. Note that, for $N=7$ and full sensing, the curve of the proposed model coincides with the unslotted version of [5]. The vertical bars indicate the standard deviation as obtained out of five simulation runs with $10^{4}$ generated packets.

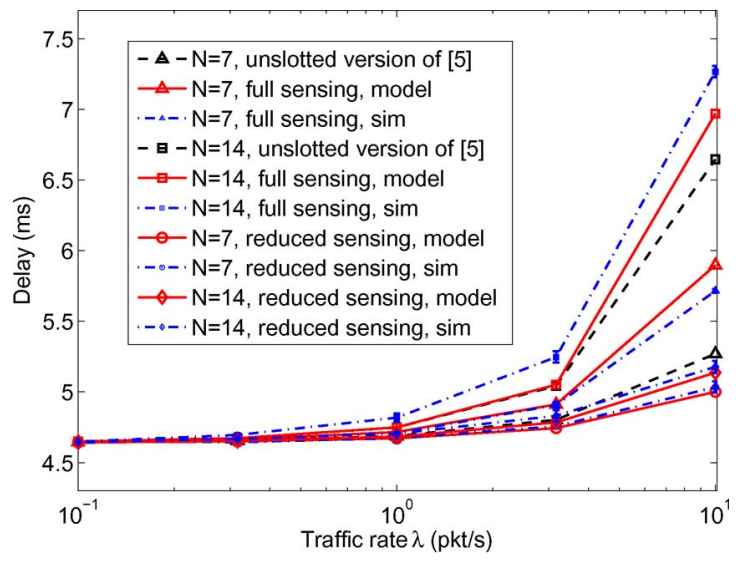

Fig. 5. Delay versus traffic rate $\lambda$ for the single-hop topology in Fig. 1(a). Full sensing and reduced sensing correspond to $\left|\Omega_{l}\right|=N$ and $\left|\Omega_{l}\right|=3$, respectively. Unslotted version of [5] refers to the single-hop model presented in [5] and experimentally validated in [9], which is limited only to the homogeneous case. The vertical bars indicate the standard deviation as obtained out of five simulation runs with $10^{4}$ generated packets.

models for high traffic and number of nodes, due to the different assumption in the derivation of the busy channel probability, as we discussed in Section IV.

In Fig. 5, we present the average delay over all the links for a single-hop network with homogeneous traffic by varying the node packet generation rate. As for the reliability, results are shown for different sizes of the network $(N=7, N=14)$ and by considering both full- and reduced-sensing capabilities. There is a good match between the simulations and our analytical model. The small gap when the traffic rate increases is due to the assumption of independent busy channel probabilities. This effect depends on the size of packets. As we discuss later in Section V-E, our model compensates for this effect, and it is a better approximation than the one in [5]. We notice here that nodes with reduced-sensing capability have a positive effect on the delay performance. This is in contrast with the effect on the

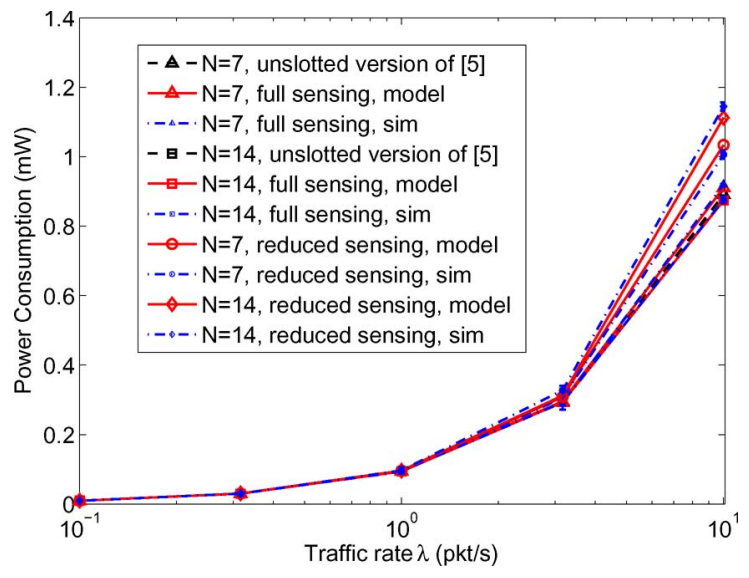

Fig. 6. Energy consumption versus traffic rate $\lambda$ for the single-hop topology in Fig. 1(a). Full sensing and reduced sensing correspond to $\left|\Omega_{l}\right|=N$ and $\left|\Omega_{l}\right|=3$, respectively. Unslotted version of [5] refers to the single-hop model presented in [5] and experimentally validated in [9], which is limited only to the homogeneous case. The vertical bars indicate the standard deviation as obtained out of five simulation runs with $10^{4}$ generated packets.

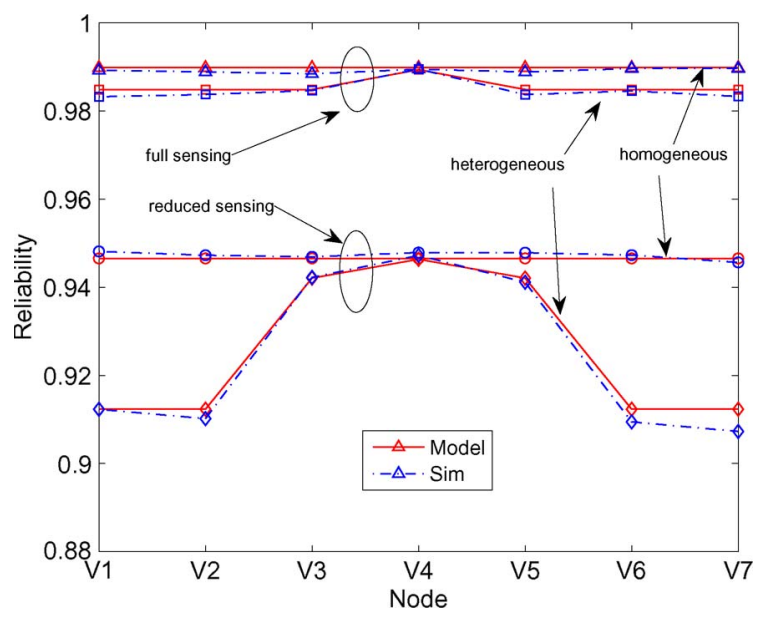

Fig. 7. Reliability of every node for the single-hop topology in Fig. 1(a). Full sensing and reduced sensing correspond to $\left|\Omega_{l}\right|=N$ and $\left|\Omega_{l}\right|=3$, respectively. In the homogeneous case, $\lambda_{l}=5 \mathrm{pkt} / \mathrm{s}$, for $l=1, \ldots, N$. In the heterogeneous case, $\lambda_{4}=20 \mathrm{pkt} / \mathrm{s}$, and $\lambda_{l}=5 \mathrm{pkt} / \mathrm{s}$, for $l \neq 4$.

reliability in Fig. 4. The reason is that the average delay is evaluated only for successfully received packets: reduced-sensing capabilities decrease the number of competitor nodes for the free channel assessment; thus, the busy channel probability is reduced, which, in turn, decreases the average delay.

In Fig. 6, the analysis of the node energy consumption is reported. We show the results for default MAC parameters with $n=1$. The energy consumption is dominated by the actual traffic for every node, because the cost for transmitting and receiving packets is much higher than the other cost components. For this reason, reduced-sensing capabilities, which influence the number of collisions, have stronger impact on the energy consumption with respect to an increase in the size of the network from $N=7$ to $N=14$ nodes. Furthermore, with $N=14$, the number of transmissions reduces with respect to $N=7$ due to the higher probability that a packet is discarded because of channel access failures.

Let us consider now the interaction between heterogeneous traffic and the reduced-sensing capability of the single-hop 


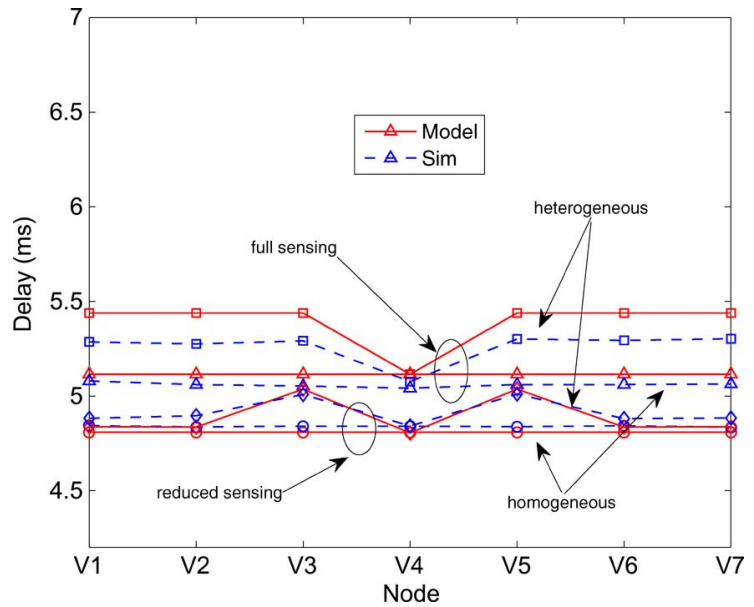

Fig. 8. Average node delay for the single-hop topology in Fig. 1(a). Full sensing and reduced sensing correspond to $\left|\Omega_{l}\right|=N$ and $\left|\Omega_{l}\right|=3$, respectively. In the homogeneous case, $\lambda_{l}=5 \mathrm{pkt} / \mathrm{s}$, for $l=1, \ldots, N$. In the heterogeneous case, $\lambda_{4}=20 \mathrm{pkt} / \mathrm{s}$, and $\lambda_{l}=5 \mathrm{pkt} / \mathrm{s}$, for $l \neq 4$.

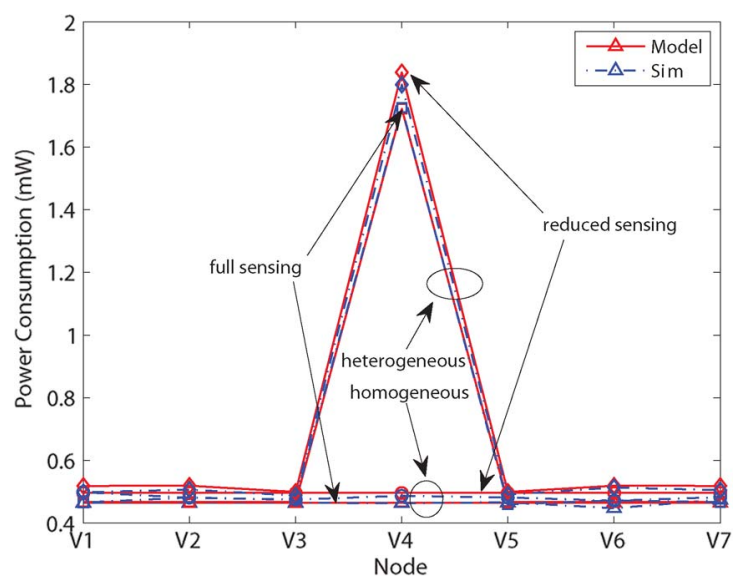

Fig. 9. Node energy consumption for the single-hop topology in Fig. 1(a). Full sensing and reduced sensing correspond to $\left|\Omega_{l}\right|=N$ and $\left|\Omega_{l}\right|=3$, respectively. In the homogeneous case, $\lambda_{l}=5 \mathrm{pkt} / \mathrm{s}$, for $l=1, \ldots, N$. In the heterogeneous case, $\lambda_{4}=20 \mathrm{pkt} / \mathrm{s}$, and $\lambda_{l}=5 \mathrm{pkt} / \mathrm{s}$, for $l \neq 4$.

topology of Fig. 1(a). In Fig. 7, we report the link reliability associated to every node. We plot analytical results and simulation of the reliability for a single-hop network with $N=7$ nodes. In the homogeneous case, every node generates the same traffic $\lambda_{l}=5 \mathrm{pkt} / \mathrm{s}, l=1, \ldots, N$. In the heterogeneous case, node $V_{4}$ generates a traffic $\lambda_{4}=20 \mathrm{pkt} / \mathrm{s}$, whereas the rest of the network has nodes with $\lambda_{l}=5 \mathrm{pkt} / \mathrm{s}$. The effect of an increased traffic of $V_{4}$ leads to a decreasing of the reliability in the rest of the network, whereas the reliability of $V_{4}$ is only marginally affected. This effect is more significant when there are reduced sensing capabilities.

In Fig. 8, we report the delay associated to every node. With full-sensing capabilities, the effect of an increased traffic in $V_{4}$ is an increase in the delay in the rest of the network, whereas the delay of $V_{4}$ is not affected. With reduced-sensing capabilities, it is interesting to notice that the delay increases only for nodes that are in the sensing range of $V_{4}$ (i.e., $V_{3}$ and $V_{5}$ ).

In Fig. 9, we show the energy consumption for every node. The increasing traffic on $V_{4}$ in the heterogeneous condition significantly affects its own energy consumption in both full-

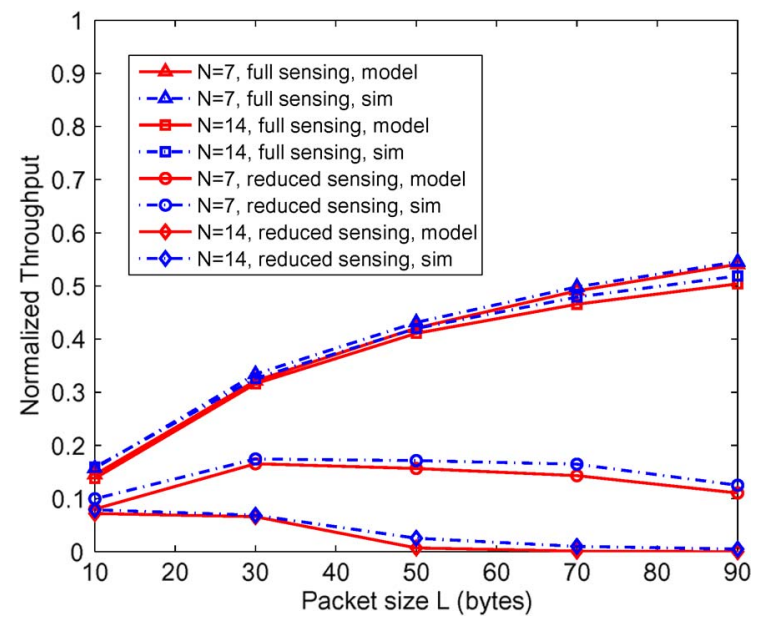

Fig. 10. Normalized throughput versus packet size $L$ for the single-hop topology in Fig. 1(a) under saturated traffic conditions. Full sensing and reduced sensing correspond to $\left|\Omega_{l}\right|=N$ and $\left|\Omega_{l}\right|=N / 2$, respectively.

and reduced-sensing capabilities, and in a lower extent, it increases the energy consumption of nodes outside its sensing range. Nodes $V_{3}$ and $V_{5}$, which are in the sensing range, are not noticeably influenced.

To show the effectiveness of the analytical model for hightraffic networks, we consider saturated conditions where packets are generated back to back at each node. Under saturated conditions, performance is typically measured in terms of normalized throughput. In Fig. 10, we report the normalized throughput by varying the packet size $L$, for a network with $N=7$ and $N=14$ under saturated conditions, for full- and reduced-sensing capabilities. Full sensing and reduced sensing correspond to $\left|\Omega_{l}\right|=N$ and $\left|\Omega_{l}\right|=N / 2$, respectively. The analytical model follows quite well the simulation. The throughput tends to increase with the packet size, and the gap between $N=$ 7 and $N=14$ is not significant for full-sensing capabilities. In the case of reduced-sensing capabilities, by increasing the packet size, the number of collisions due to hidden nodes increases, and for large $L$, the throughput decreases. This effect is more evident as the number of nodes increases to $N=14$ due to the higher number of hidden nodes.

We now consider large-scale networks and validate the approximate model presented in Section IV-G. In Fig. 11, we show the average reliability for a single-hop network by varying the number of nodes $N$. We plot analytical results and simulation of the reliability by considering $\lambda_{l}=[1,5] \mathrm{pkt} / \mathrm{s}$, with full and reduced carrier-sensing capabilities. There is a good agreement between the approximate model and the simulations. As the traffic increases, the performance significantly degrades with the number of nodes.

We conclude that heterogeneous traffic conditions and hidden terminals have significant and complex effects on each one of the performance indicators (reliability, delay, and energy consumption), and the effects are well predicted by our model both for small- and large-scale networks. In particular, dominant nodes, i.e., nodes with heavy forwarded traffic load, negatively affect the performance of the other nodes of the network. In the next section, we show how routing decisions are 


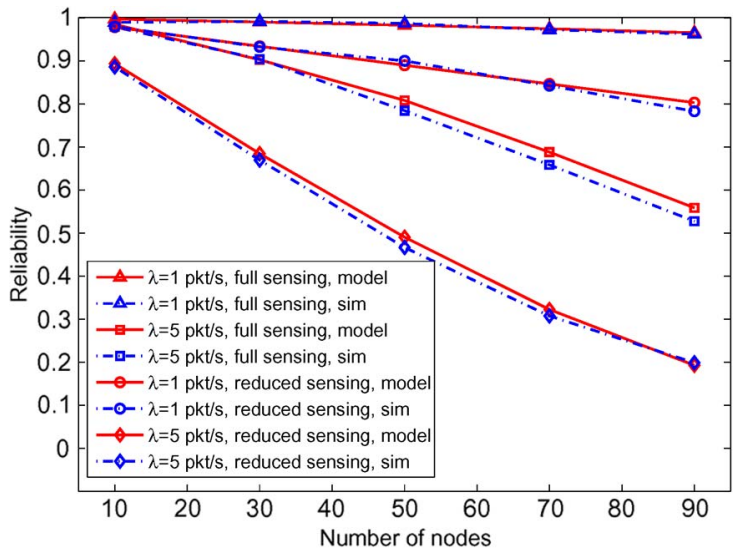

Fig. 11. Reliability versus number of nodes $N$ for the single-hop topology in Fig. 1(a). Full sensing and reduced sensing correspond to $\left|\Omega_{l}\right|=N$ and $\left|\Omega_{l}\right|=N / 2$, respectively.

influenced by traffic, carrier-sensing range, and performance indicators in multi-hop networks.

\section{B. Multi-hop Network}

In this section, we validate the analysis for multi-hop networks proposed in Section IV-F. First, we consider the topology of Fig. 1(b). Without loss of generality, we assume that every node generates the same traffic, but the forwarded traffic from every node varies as a consequence of the multi-hop routing. We focus on two cases, which we denote by Path 1 and Path 2 . In Path 1 , we analyze the end-to-end reliability when node $V_{7}$ routes all its packets along path $V_{4}-V_{1}$ to root node $V_{0}$. In Path 2, $V_{7}$ forwards its packets along path $V_{6}-V_{3}$. We also distinguish between noninterfering and interfering paths. In the former case, nodes along the two paths (Path 1 and Path 2) do not sense each other, with the exception of the last hop to $V_{0}$. In the latter case, interference among path is determined by letting the carrier-sensing range of nodes in a path include nodes in the other path, with exception of the last hop to $V_{0}$. The list of nodes in the carrier-sensing range of each node for the two cases is reported in Table II.

In Fig. 12, we report the end-to-end reliability from node $V_{7}$ to the root node $V_{0}$, and the average end-to-end reliability of the whole network, by varying the node packet generation rate, when the two paths are noninterfering. From both analytical results and simulations, Path 2 outperforms Path 1 in terms of reliability, from both a single node and a network perspective, and the difference increases as the traffic increases. For $\lambda=$ $10 \mathrm{pkt} / \mathrm{s}$, the gap between the two paths is $7 \%-10 \%$. In fact, Path 2 has lower forwarded traffic load than Path 1.

Fig. 13 shows the end-to-end reliability of node $V_{7}$, and the average network reliability for analytical model and simulations when Path 1 and Path 2 are interfering. Compared to Fig. 12, the result is different, and the best performance in terms of reliability is now on Path 1 , from both a node and a network perspective. The gap increases with the traffic, and for $\lambda=$ $10 \mathrm{pkt} / \mathrm{s}$, Path 1 outperforms Path 2 of $7 \%-10 \%$. Nodes in Path 1 turn out to be dominant in terms of traffic load and affect negatively the performance of nodes in Path 2 because the reliability of a contention access scheme increases as the

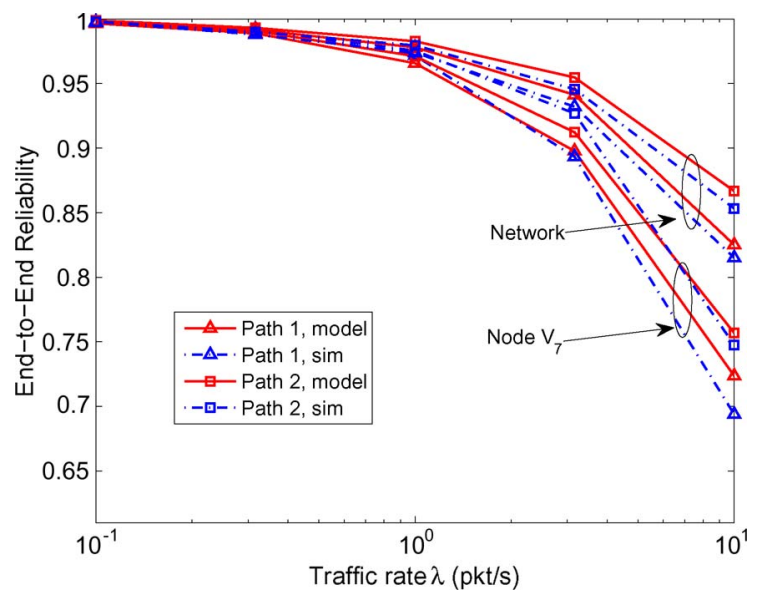

Fig. 12. End-to-end reliability for noninterfering paths in the multi-hop topology in Fig. 1(b). Path 1 is $V_{7}-V_{4}-V_{1}-V_{0}$ when link $V_{7}-V_{6}$ is disabled. Path 2 is $V_{7}-V_{6}-V_{3}-V_{0}$ when link $V_{7}-V_{4}$ is disabled. Notice the different scale on the $y$-axis compared with the link reliability in Figs. 4 and 7.

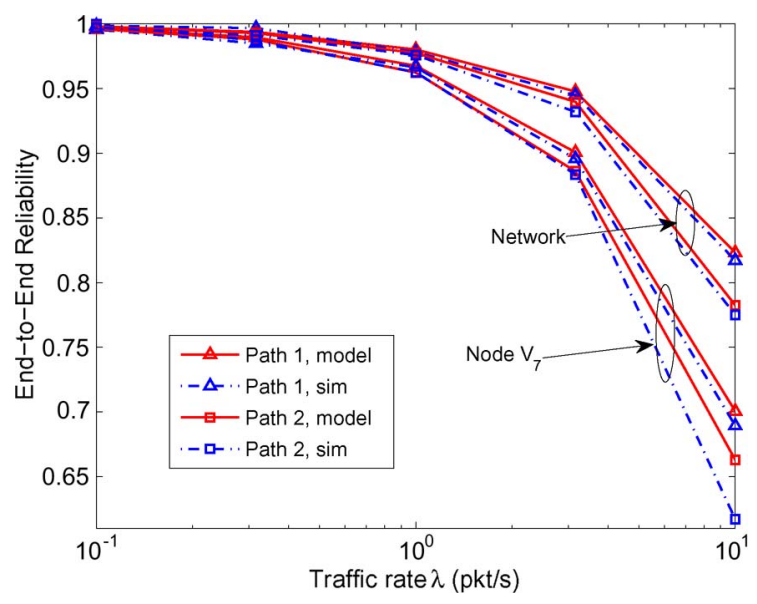

Fig. 13. End-to-end reliability for interfering paths in the multi-hop topology in Fig. 1(b). Path 1 is $V_{7}-V_{4}-V_{1}-V_{0}$ when link $V_{7}-V_{6}$ is disabled. Path 2 is $V_{7}-V_{6}-V_{3}-V_{0}$ when link $V_{7}-V_{4}$ is disabled.

average number of contenders and hidden terminals in each time unit are reduced. Consider a routing metric based only on the maximization of the end-to-end reliability. If there is a strong interference in the network, the routing decision leads to unbalanced distribution of traffic load, because the routing layer forces the forwarded traffic flow to more dominant nodes, so that the average number of contenders in each time unit is lower. If not taken into account, this phenomenon may be catastrophic for the network and cause stability issues when considering limited node buffer size and energy constraints. On the other side, when stability and load balancing are not critical, dominant paths can be used to improve the end-to-end performance.

In Figs. 14 and 15, the end-to-end delay from node $V_{7}$ to the root node $V_{0}$ is shown for noninterfering paths and interfering paths, respectively. Path 2 outperforms Path 1 when there is no interference among paths. For $\lambda=10 \mathrm{pkt} / \mathrm{s}$, the gap between the two paths is $5 \%-7 \%$. Path 1 has a lower delay when the two paths are interfering. For $\lambda=10 \mathrm{pkt} / \mathrm{s}$, the gap between the two paths is $3 \%-5 \%$. Similarly to the reliability analysis, 


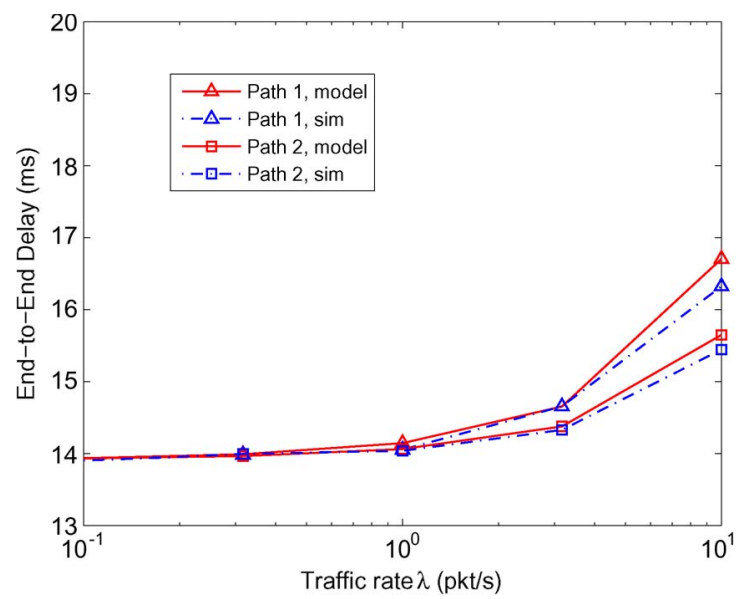

Fig. 14. End-to-end delay for noninterfering paths in the multi-hop topology in Fig. 1(b). Path 1 is $V_{7}-V_{4}-V_{1}-V_{0}$ when link $V_{7}-V_{6}$ is disabled. Path 2 is $V_{7}-V_{6}-V_{3}-V_{0}$ when link $V_{7}-V_{4}$ is disabled.

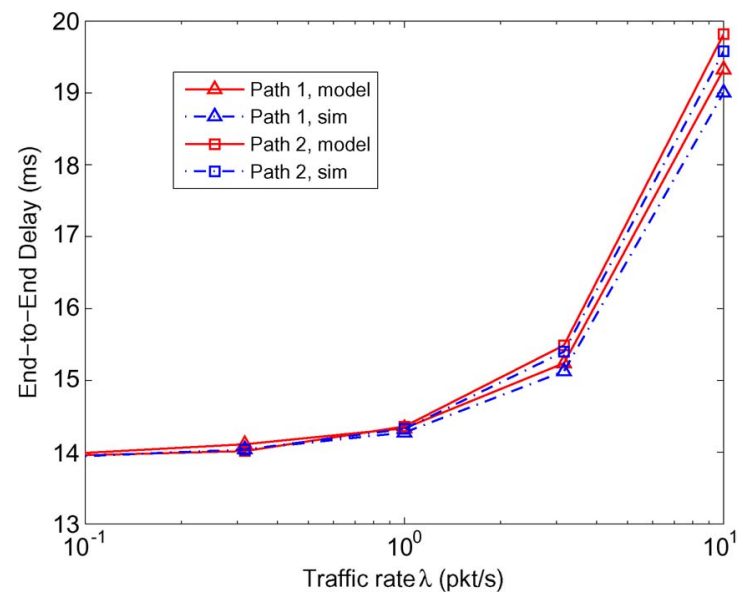

Fig. 15. End-to-end delay for interfering paths in the multi-hop topology in Fig. 1(b). Path 1 is $V_{7}-V_{4}-V_{1}-V_{0}$ when link $V_{7}-V_{6}$ is disabled. Path 2 is $V_{7}-V_{6}-V_{3}-V_{0}$ when link $V_{7}-V_{4}$ is disabled.

if the routing metric is based only on the minimization of the end-to-end delay, and there is path interference, the result is an unbalanced traffic distribution toward dominant nodes. Once again, if not correctly taken into account, this could lead to negative effects on the network.

In the following, we show simulation results for the multihop topology in Fig. 1(c). We consider multiple end devices $\left(V_{4}, V_{5}, V_{6}\right.$, and $\left.V_{7}\right)$ that may decide to route their packets either through nodes $V_{1}, V_{2}$, or $V_{3}$ to the destination $V_{0}$. The routing decision is based on the end-to-end reliability. We consider a dominant node $V_{2}$ that generates $\lambda_{2}=20 \mathrm{pkt} / \mathrm{s}$, whereas the rest of the network operates with traffic generation rate $\lambda_{i}=$ $5 \mathrm{pkt} / \mathrm{s}$. Similarly to the previous configuration, we distinguish between noninterfering and interfering paths. In the former case, the carrier-sensing range of end devices includes only the candidate parents. In the latter case, interference among path is determined by letting the carrier-sensing range of all end devices include all the other nodes in the network. The list of nodes in the carrier-sensing range of each node for the two cases is reported in Table II. In Fig. 16, we show the resulting time evolution of the parent selection for each end device in the

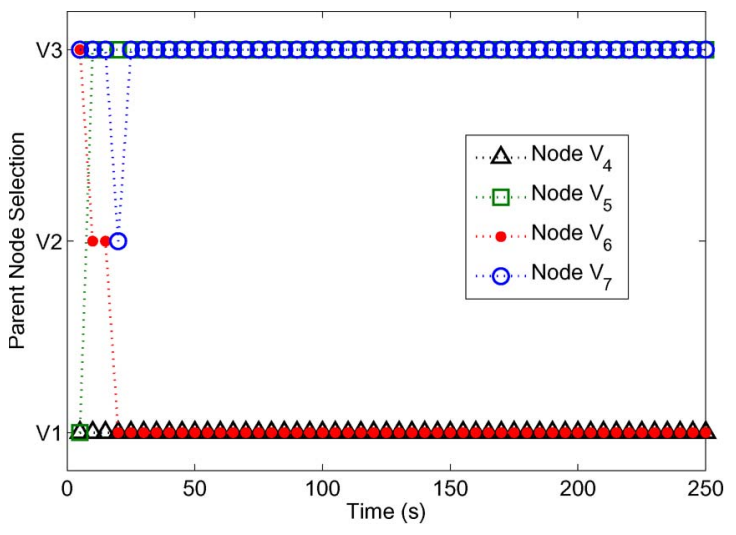

Fig. 16. Parent node selection versus time for noninterfering paths in the multi-hop topology in Fig. 1(c).

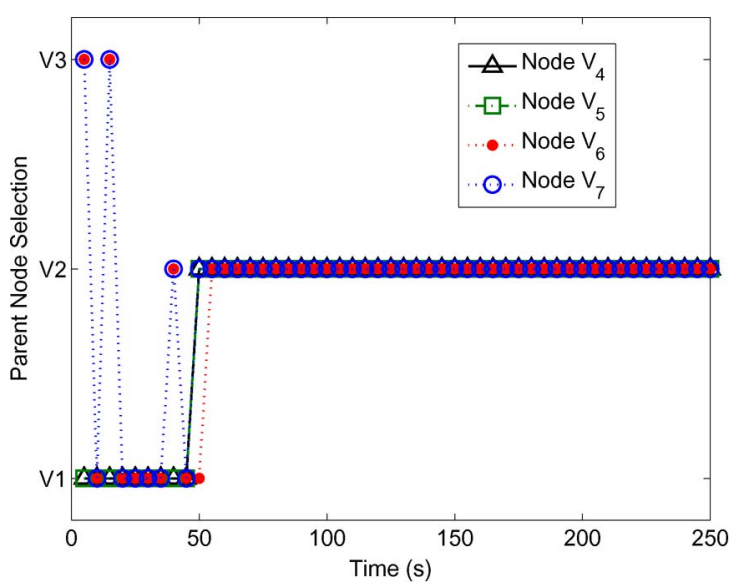

Fig. 17. Parent node selection versus time for interfering paths in the multihop topology in Fig. 1(c).

network for noninterfering paths. The end devices start from a random initial condition and explore the various routing paths to determine the next-hop node that guarantees the highest endto-end reliability. Nodes use sliding windows to average the reliability in each iteration. In the case of noninterfering paths, nodes tend to distribute the traffic toward node $V_{1}$ and $V_{3}$, avoiding the dominant node $V_{2}$. In Fig. 17, we show the time evolution of the parent selection when paths are interfering. In this situation, nodes tend to concentrate the traffic toward the dominant node $V_{2}$. This result is consistent with the previous analysis for a single end device. The time evolution of the average end-to-end reliability is shown in Fig. 18 for both noninterfering and interfering paths, whereas a reference, i.e., the end-to-end reliability computed by the analytical model, is reported.

\section{Effect of Imperfect Carrier Sensing}

The assumption of perfect carrier sensing plays a critical role in understanding the fundamental limitations of the IEEE 802.15.4 protocol. However, in the unslotted mechanism of the IEEE 802.15.4, MAC nodes are not time synchronized. In this section, we analyze the performance of CSMA/CA algorithm in the presence of carrier-sensing errors. We consider the typical two types of carrier-sensing errors, i.e., false negative and false 


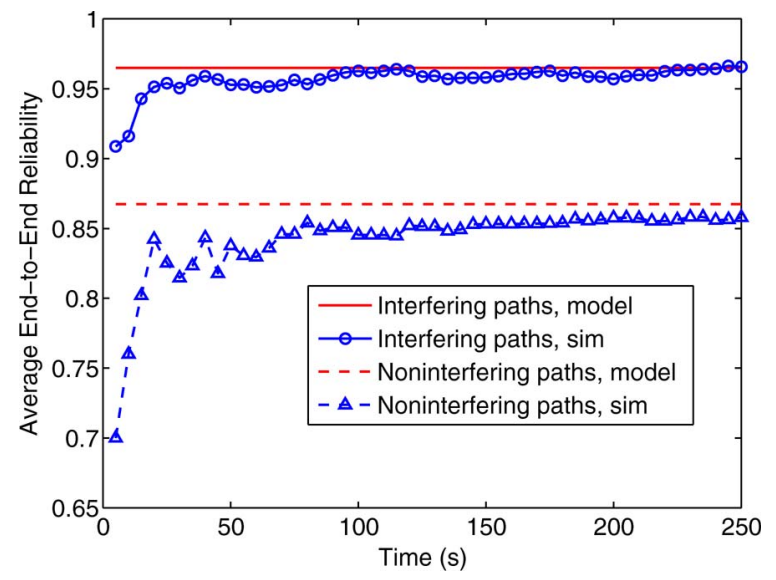

Fig. 18. Average end-to-end reliability of $V_{4}, V_{5}, V_{6}$, and $V_{7}$ versus time for interfering and noninterfering paths in the multi-hop topology in Fig. 1(c).

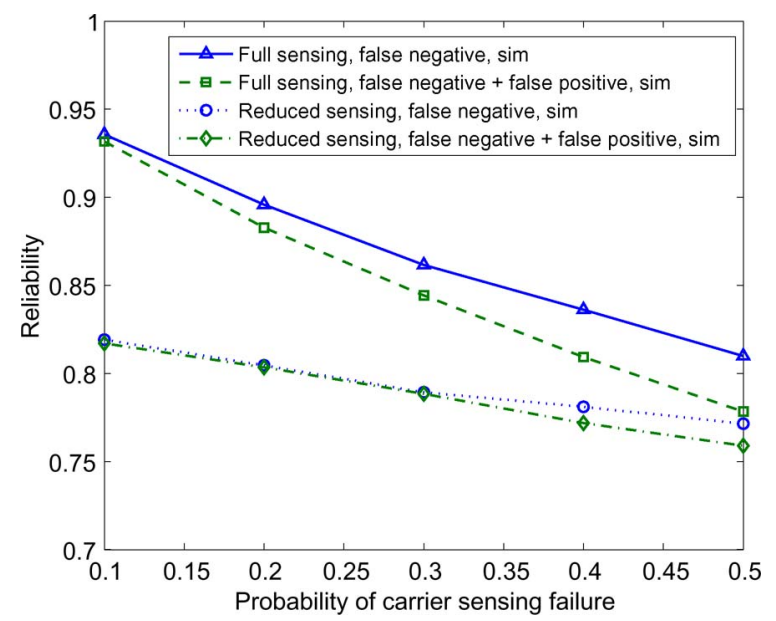

Fig. 19. Reliability versus probability of carrier-sensing failure for the singlehop topology in Fig. 1(a), with $N=7$, and $\lambda_{l}=10 \mathrm{pkt} / \mathrm{s}$, for $l=1, \ldots, N$. Full sensing and reduced sensing correspond to $\left|\Omega_{l}\right|=N$ and $\left|\Omega_{l}\right|=3$, respectively. Note that "false negative" and "false negative + false positive" refer to the consideration of the false negative event and the combined event of the false negative and positive failure for the simulation setup, respectively.

positive. Their impact on the system performance is analyzed by using simulation results. A false negative failure happens when the carrier sensing incorrectly detects that the medium is idle when it is actually busy. A false positive event occurs when a busy state is reported when the medium is idle. We model carrier-sensing failures by independent Bernoulli trials with success probability $1-p_{f}$, where $0 \leq p_{f} \leq 1$. The independence of the trial results is assumed to be over all the links and the attempts.

We consider the single-hop network in Fig. 1(a) with $N=7$, with both full- and reduced-sensing capabilities. In Figs. 19 and 20, we report the reliability and average packet delay, respectively, as a function of the probabilities of the carrier sensing error. Note that "false negative" and "false negative + false positive" refer to the consideration of the false negative event and the combined event of the false negative and positive failure for the simulation setup, respectively. The false negative and positive failures decrease the system performance in terms of reliability. The effect of the false positive failure on the reliability is more evident as the failure probability increases. The false negative failure slightly decreases the delay of suc-

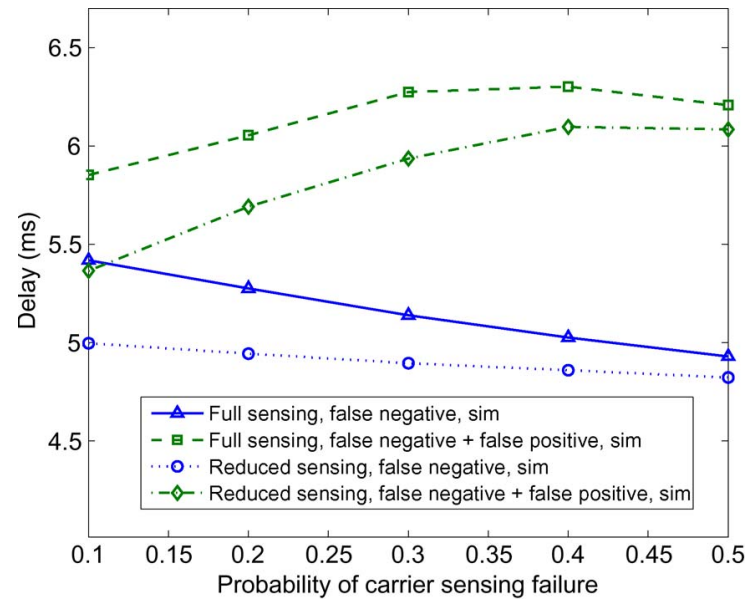

Fig. 20. Delay versus probability of carrier-sensing failure for the singlehop topology in Fig. 1(a), with $N=7$, and $\lambda_{l}=10 \mathrm{pkt} / \mathrm{s}$, for $l=1, \ldots, N$. Full sensing and reduced sensing correspond to $\left|\Omega_{l}\right|=N$ and $\left|\Omega_{l}\right|=3$, respectively. Note that "false negative" and "false negative + false positive" refer to the consideration of the false negative event and the combined event of the false negative and positive failure for the simulation setup, respectively.

TABLE III

Computation Time of Analytical Model and Simulations

\begin{tabular}{|c|c|c|c|c|}
\hline$\lambda$ & $N$ & Model & Approx & Simulations \\
\hline \multirow{3}{*}{$0.1 \mathrm{pkt} / \mathrm{s}$} & 7 & $6.8 \mathrm{~s}$ & $0.4 \mathrm{~s}$ & $2.5 \cdot 10^{5} \mathrm{~s}$ \\
& 14 & $1810 \mathrm{~s}$ & $0.6 \mathrm{~s}$ & $4.8 \cdot 10^{5} \mathrm{~s}$ \\
& 50 & - & $9.3 \mathrm{~s}$ & $2.6 \cdot 10^{6} \mathrm{~s}$ \\
\hline \multirow{3}{*}{$1 \mathrm{pkt} / \mathrm{s}$} & 7 & $7.0 \mathrm{~s}$ & $0.5 \mathrm{~s}$ & $2.5 \cdot 10^{4} \mathrm{~s}$ \\
& 14 & $2262 \mathrm{~s}$ & $0.6 \mathrm{~s}$ & $4.8 \cdot 10^{4} \mathrm{~s}$ \\
& 50 & - & $9.2 \mathrm{~s}$ & $2.8 \cdot 10^{5} \mathrm{~s}$ \\
\hline \multirow{3}{*}{$10 \mathrm{pkt} / \mathrm{s}$} & 7 & $8.2 \mathrm{~s}$ & $0.4 \mathrm{~s}$ & $2530 \mathrm{~s}$ \\
& 14 & $2317 \mathrm{~s}$ & $0.6 \mathrm{~s}$ & $4910 \mathrm{~s}$ \\
& 50 & - & $9.5 \mathrm{~s}$ & $2.9 \cdot 10^{4} \mathrm{~s}$ \\
\hline
\end{tabular}

cessfully received packets since it reduces the average number of backoffs in the network. The false positive failure affects directly the average number of backoffs, and it increases the average delay. For large values of false positive probability, packets start to be dropped due to the number of backoffs and the delay starts decreasing. If the carrier-sensing failure probability is small $\left(p_{f}<0.2\right)$, the effect of the imperfect carrier sensing is not critical for the reliability and delay. Hence, we argue that our model predicts well the performance of the network for small false negative and false positive probabilities.

\section{Computation Time of Analytical Model and Simulations}

The accurate Markov chain analysis considers the solution of a system of nonlinear equations, which scales with the number of links. For large dense networks with high connectivity, this model may be computationally expensive. Therefore, we have introduced approximated model equations in Section IV-G.

Table III compares the computation time of both analytical model and simulations as a function of the number of nodes and traffic rate. The system of nonlinear equations is solved using the common trust-region dogleg algorithm [25]. The Monte Carlo simulations comprise of five runs of $10^{4}$ packets generated. The computation time for the analytical model is significantly reduced with respect to the simulation time. The simulation time increases as the traffic rate decreases (fewer 


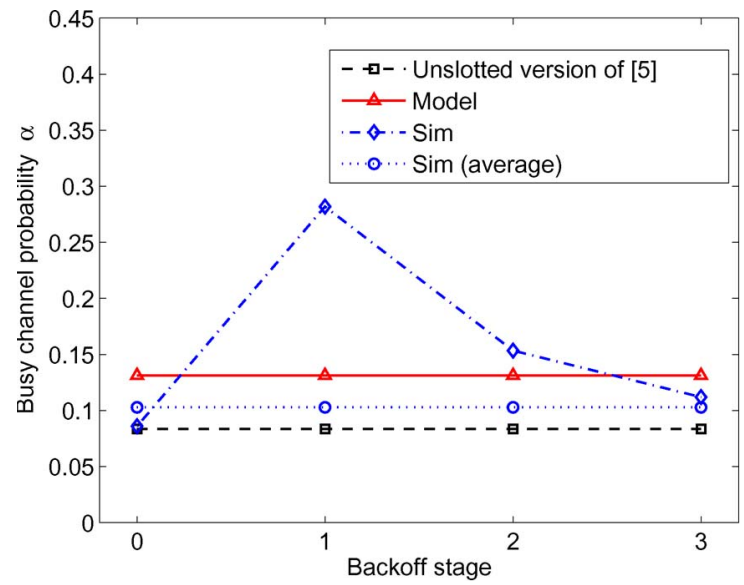

Fig. 21. Busy channel probability versus backoff stage for the single-hop topology in Fig. 1(a), with $N=7$, and $\lambda_{l}=5 \mathrm{pkt} / \mathrm{s}$, for $l=1, \ldots, N$, with full-sensing capabilities. Unslotted version of [5] refers to the single-hop model presented in [5] and experimentally validated in [9], which is limited only to the homogeneous case. The curve "sim (average)" represents the average busy channel probability independently of the backoff stage.

packets are generated per time unit) and as the number of nodes increases. The accurate analytical model computation time significantly depends on the number of nodes. However, the number of nodes in an one-hop neighborhood is typically up to ten, according to the IETF ROLL routing requirements for urban, industrial, and home environments [2]. When the approximate model is used, the computation time also remains limited for large networks.

\section{E. Model Limitations}

In this section, we investigate the limitations of the model for IEEE 802.15.4 multi-hop networks. The basic model assumption is the independent busy channel probability $\alpha_{l}$ along the backoff stages of the Markov chain. In practice, this is realistic for many situations but not for all. In [12], it is shown that this assumption has an impact on the delay and power consumption, mainly for small scale networks. In Fig. 21, we report the average busy channel probability in different backoff stages of the chain (i.e., $i=0 i=1, i=2$ ). We consider $N=7$ nodes with no hidden terminals, MAC parameters $m_{0}=3, m=4$, $m_{b}=7, n=0$, traffic rate $\lambda_{l}=5 \mathrm{pkt} / \mathrm{s}$ for $l=1, \ldots, N$, packet size $L=7$, and ACK size $L_{\text {ack }}=2$, and we compare results from the analytical model with Monte Carlo simulations. As a reference, we report the busy channel probability obtained from the single-hop homogeneous model with full-sensing capabilities presented in [5]. The simulation results show a significant increase in the busy channel probability between the initial backoff stage $(i=0)$ and the second backoff stage $(i=1)$. Then, the probability decreases in the following backoff stages to agree with the analysis. The reason of this increase is due to the transmission time (of packets plus ACKs), which is not negligible with respect to the backoff time. If the channel is busy after the initial backoff, there is a certain probability that the same transmission is still ongoing after the second backoff. This probability reduces as the backoff increases in the following stages. In [5], as in the previous literature, the busy channel probability is constant for the entire network. As we see in Fig. 21, the value predicted by the theoretical model in [5] well approximates the busy channel probability of the simulation in the initial backoff $(i=0)$, but it underestimates the average busy channel probability. Our multi-hop model assumes that, for every node $V_{l}, \alpha_{l}$ is computed independently of the busy channel probability of other nodes. This analytical approach tends to overestimate the busy channel probability in the initial backoff stage, but it compensates for the increase in $\alpha_{l}$ in the following backoff stages.

In [6], a derivation of the busy channel probability for the different backoff stages is introduced. However, the system scenario is different from our model scenario. In fact, the network topology in [6] considers a single-hop star network, in which nodes transmits packets only upon reception of a query from the personal-area network coordinator. Therefore, the carrier-sensing mechanism is synchronized for all nodes, and the number of contending nodes at the beginning of the first backoff is supposedly known. By contrast, in our system scenario, nodes are allowed to generate traffic with a random Poisson distribution at different rates, which is more general and greatly complicates the analysis. The number of contending nodes that a transmitting node encounters when it starts the first backoff procedure cannot be determined a priori, and those nodes that are contending are not necessarily in their first backoff. Moreover, the presence of retransmitted packets complicates the analysis. Therefore, we believe that the analytical derivation of the impact of packet size on the busy channel probability in the different backoff stages in our model scenario is a formidable task. However, as shown in the previous sections, an approximation by a busy channel probability that is independent of the backoff stage is accurate enough to derive the network reliability, delay, and energy consumption.

\section{CONCLUSION}

In this paper, we have proposed a novel analysis of singlehop and multi-hop networks using the unslotted IEEE 802.15.4 MAC protocol. We have introduced an accurate analytical model that includes the important features of multi-hop networks, such as heterogeneous distribution of traffic, hidden terminal nodes, and queue length. An approximate model has also been introduced, and it has been validated for large networks up to about hundred nodes. We have shown mutual influence between routing decisions and MAC performance in terms of reliability, delay, and load balancing.

The goal of this paper was not to provide routing solutions but to show the effectiveness of our proposed model in capturing the interaction between MAC and routing. Therefore, based on this model, our future work will investigate optimal routing metrics for IEEE 802.15.4 multi-hop networks.

\section{APPENDIX A}

\section{QUEUEING MODEL FOR THE MARKOV CHAIN IN FIG. 2}

In this section, we derive the probabilities of having a new packet to send at MAC layer, in the different stages of the Markov chain. We consider four different situations: 1) probability of generation of a new packet after an idle unit time $q_{l}$; 2) probability that the node queue is not empty after a packet 
has been successfully sent $q_{\mathrm{succ}, l}=1-p_{l, 0}^{s} ; 3$ ) probability that the node queue is not empty after a packet has been discarded due to channel access failure $q_{\mathrm{cf}, l}=1-p_{l, 0}^{m}$; and 4) probability that the node queue is not empty after a packet has been discarded due to the retry limit $q_{\mathrm{cr}, l}=1-p_{l, 0}^{n}$. By assuming generation of packets with Poisson distribution at rate $\lambda_{l}$, the probability of generation of a new packet after an idle unit time can be easily derived as $q_{l}=1-\exp \left(-\lambda_{l} / S_{b}\right)$. The other terms are derived in the following.

If we assume limited buffer size $B$, the packet queue in the device buffer can be modeled as a $M / G / 1 / K$ queueing system. Denoting as $a_{k}$ the probability to have $k$ packets arrivals into the buffer of a node during the service time, its probabilitygenerating function (PGF) can be expressed as function of the PGF of the service time $\mathcal{T}_{l}(z)$ as [24]

$$
a_{k}=\left.\frac{1}{k !} \frac{d^{k} \mathcal{T}_{l}\left(1-q_{l}+q_{l} z\right)}{d z^{k}}\right|_{z=0} .
$$

The steady-state probability $p_{l, k}$ that there are $k$ packets in the buffer of node $V_{l}$ after a packet transmission attempt is given by solving the following system in a recursive manner [3]:

$$
\left\{\begin{array}{l}
p_{l, k}=p_{l, 0} a_{k}+\sum_{j=1}^{k+1} p_{l, j} a_{k-j+1}, \quad 0 \leq k \leq B-2 \\
p_{l, B-1}=p_{l, 0} \sum_{k=B-1}^{\infty} a_{k}+\sum_{j=1}^{B-1} p_{l, j} \sum_{k=B-1}^{\infty} a_{k} .
\end{array}\right.
$$

By inserting the expression of the PGF of the service time for successful transmission $\mathcal{T}_{\mathrm{tx}, l}$, failure due to the maximum number of backoffs $\mathcal{T}_{\text {cf }, l}$ and failure due to retry limit $\mathcal{T}_{\text {cr }, l}$ into (28), it is possible to derive the probabilities of empty queue $p_{l, 0}^{s}, p_{l, 0}^{m}$, and $p_{l, 0}^{n}$, respectively.

The delay distribution of IEEE 802.15.4 MAC with ACK and retransmission is studied in [26], and the PGFs are derived for the slotted mechanism. The derivation for the Markov chain in Fig. 2 follows similar steps. However, the derivation of the PGFs for each node is unpractical and computationally expensive. In case the average queue length is lower than the buffer size $B$, a good approximation of the empty queue probability can be obtained by using a $M / G / 1$ model [13]. For Poisson arrivals with rate $\lambda_{l}$, we obtain

$$
p_{l, 0}^{s}=1-\lambda_{l} \mathbb{E}\left\{D_{l}^{s}\right\}
$$

where the average service time is computed in Section IV-D.

\section{APPENDIX B}

\section{PROOF OF PROPOSITION 4.1}

First, we compute the stationary distribution of the Markov chain in Fig. 2. The transition probabilities of the chain are

$$
\begin{aligned}
& \operatorname{Pr}[i, k, j \mid i, k+1, j]=1, \quad \text { for } k \geq 0 \\
& \operatorname{Pr}[i, k, j \mid i-1,0, j]=\frac{\alpha_{l}}{W_{i}}, \quad \text { for } i \leq m \\
& \operatorname{Pr}[0, k, j \mid i, 0, j-1]=\frac{\left(1-\alpha_{l}\right) P_{\text {coll }, l}}{W_{0}}, \quad \text { for } j \leq n \\
& \operatorname{Pr}[\text { idle } \mid i, 0, j]=\left(1-q_{\text {succ }, l}\right)\left(1-P_{\text {coll }, l}\right) \alpha_{l}, \\
& \text { for } i<m, j<n
\end{aligned}
$$

$$
\begin{array}{lr}
\operatorname{Pr}[\text { idle } \mid m, 0, j]=\left(1-q_{\mathrm{cf}, l}\right) \alpha_{l}, & \text { for } j<n \\
\operatorname{Pr}[\text { idle } \mid i, 0, n]=\left(1-q_{\mathrm{cr}, l}\right)\left(1-\alpha_{l}\right), & \text { for } i<m \\
\operatorname{Pr}[\text { idle } \mid m, 0, n]=\left(1-q_{\mathrm{cr}, l}\right)\left(1-\alpha_{l}\right)+\left(1-q_{\mathrm{cf}, l}\right) \alpha_{l} \\
\operatorname{Pr}[0, k, 0 \mid \text { idle }]=\frac{q_{l}}{W_{0}}, \quad \text { for } k \leq W_{0}-1 .
\end{array}
$$

Equation (31) is the decrement of backoff counter, which happens with probability 1 . Equation (32) represents the probability of finding busy channel in CCA and of choosing a state uniformly in the next backoff stage. Equation (33) gives the probability of unsuccessful transmission after finding a clear channel, and a node selects uniformly a state in the next retransmission. Equations (34)-(36) represent the probability of going back to the idle stage due to success, channel access failure, and retry limit, respectively. Equation (37) is the probability of going back to the idle stage at backoff counter $m$ and retransmission stage $n$. Equation (38) models the probability of going back to the first backoff stage from the idle stage.

Owing to the chain regularities and (31)-(38), we have

$$
b_{i, k, j}^{(l)}=\frac{W_{i}-k}{W_{i}} b_{i, 0, j}^{(l)}
$$

where

$$
W_{i}= \begin{cases}2^{i} W_{0}, & i \leq m_{b}-m_{0} \\ 2^{m_{b}-m_{0}} W_{0}, & i>m_{b}-m_{0} .\end{cases}
$$

From (32), for $i \leq m$, we obtain

$$
b_{i, 0, j}^{(l)}=\alpha_{l}^{i} b_{0,0, j}^{(l)} .
$$

From (33), $b_{0,0, j}^{(l)}$ is rewritten as follows:

$$
b_{0,0, j}^{(l)}=\left(\left(1-\alpha_{l}\right) P_{\mathrm{coll}, l} \sum_{i=0}^{m} \alpha_{l}^{i}\right)^{j} b_{0,0,0}^{(l)} .
$$

By the normalization condition, we know that

$$
\begin{array}{r}
\sum_{i=0}^{m} \sum_{k=0}^{W_{i}-1} \sum_{j=0}^{n} b_{i, k, j}^{(l)}+\sum_{j=0}^{n}\left(\sum_{k=0}^{L_{s}-1} b_{-1, k, j}^{(l)}+\sum_{k=0}^{L_{c}-1} b_{-2, k, j}^{(l)}\right) \\
+b_{\text {idle }}^{(l)}=1 .
\end{array}
$$

We next derive the expressions of each term in (42). From (39) (40) (41), we have

$$
\begin{aligned}
\sum_{i=0}^{m} & \sum_{k=0}^{W_{i}-1} \sum_{j=0}^{n} b_{i, k, j}^{(l)}=\sum_{i=0}^{m} \sum_{j=0}^{n} \frac{W_{i}+1}{2} \alpha_{l}^{i} b_{0,0, j}^{(l)} \\
= & \left\{\begin{array}{c}
\frac{b_{0,0,0}^{(l)}}{2}\left(\frac{1-\left(2 \alpha_{l}\right)^{m+1}}{1-2 \alpha_{l}} W_{0}+\frac{1-\alpha_{l}^{m+1}}{1-\alpha_{l}}\right) \frac{1-y_{l}^{n+1}}{1-y_{l}}, \\
\quad \text { if } m \leq \bar{m}=m_{b}-m_{0} \\
\frac{b_{0,0,0}^{(l)}}{2}\left(\frac{1-\left(2 \alpha_{l}\right)^{\bar{m}+1}}{1-2 \alpha_{l}} W_{0}+\frac{1-\alpha_{l}^{\bar{m}+1}}{1-\alpha_{l}}\right. \\
\left.\quad+\left(2^{m_{b}}+1\right) \alpha_{l}^{\bar{m}+1} \frac{1-\alpha_{l}^{m-\bar{m}}}{1-\alpha_{l}}\right) \frac{1-y_{l}^{n+1}}{1-y_{l}}, \quad \text { otherwise }
\end{array}\right.
\end{aligned}
$$

where $y_{l}=P_{\text {coll }, l}\left(1-\alpha_{l}^{m+1}\right)$. 
Similarly

$$
\begin{aligned}
& \sum_{j=0}^{n}\left(\sum_{k=0}^{L_{s}-1} b_{-1, k, j}^{(l)}+\sum_{k=0}^{L_{c}-1} b_{-2, k, j}^{(l)}\right) \\
& =\left(L_{s}\left(1-P_{\mathrm{coll}, l}\right)+L_{c} P_{\mathrm{coll}, l}\right)\left(1-\alpha_{l}^{m+1}\right) \frac{1-y_{l}^{n+1}}{1-y_{l}} b_{0,0,0}^{(l)} .
\end{aligned}
$$

By considering that the successful transmission and the failure events are due to the limited number of backoff stages $m$ and the retry limit $n$, the idle state probability is

$$
\begin{aligned}
b_{\mathrm{idle}}^{(l)}= & \left(1-q_{l}\right) b_{\mathrm{idle}}^{(l)}+\left(1-q_{\mathrm{cf}, l}\right) \sum_{j=0}^{n} \alpha_{l} b_{m, 0, j}^{(l)} \\
& +\left(1-q_{\mathrm{cr}, l}\right) \sum_{i=0}^{m} P_{\mathrm{coll}, l}\left(1-\alpha_{l}\right) b_{i, 0, n}^{(l)} \\
& +\left(1-q_{\mathrm{succ}, l}\right) \sum_{i=0}^{m} \sum_{j=0}^{n}\left(1-P_{\mathrm{coll}, l}\right)\left(1-\alpha_{l}\right) b_{i, 0, j}^{(l)} \\
= & \frac{1}{q_{l}}\left[\left(1-q_{\mathrm{cf}, l}\right) \frac{\alpha_{l}^{m+1}\left(1-y_{l}^{n+1}\right)}{1-y_{l}}+\left(1-q_{\mathrm{cr}, l}\right) y_{l}^{n+1}\right. \\
& \left.\quad+\left(1-q_{\mathrm{succ}, l}\right)\left(1-P_{\mathrm{coll}, l}\right) \frac{\left(1-\alpha_{l}^{m+1}\right)\left(1-y_{l}^{n+1}\right)}{1-y_{l}}\right] \\
& \quad \times b_{0,0,0}^{(l)} .
\end{aligned}
$$

Note that (43)-(45) give the state values $b_{i, k, j}^{(l)}$ as a function of $b_{0,0,0}^{(l)}$. By replacing (43)-(45) in the normalization condition given by (42), we obtain $b_{0,0,0}^{(l)}$ in (3).

As a last step, we can derive $\tau_{l}$ by summing up the probabilities of being in the generic sensing stage $b_{i, 0, j}^{(l)}$, i.e.,

$$
\tau_{l}=\sum_{i=0}^{m} \sum_{j=0}^{n} b_{i, 0, j}^{(l)}=\left(\frac{1-\alpha_{l}^{m+1}}{1-\alpha_{l}}\right)\left(\frac{1-y_{l}^{n+1}}{1-y_{l}}\right) b_{0,0,0}^{(l)} .
$$

\section{REFERENCES}

[1] IEEE 802.15.4 Wireless Medium Access Control (MAC) and Physical Layer (PHY) Specifications for Low-Rate Wireless Personal Area Networks (WPANs), IEEE Std. 802.15.4, 2006.

[2] Routing over low power and lossy networks (ROLL), 2011. [Online]. Available: http://www.ietf.org/dyn/wg/charter/roll-charter.html

[3] J. Miši, S. Shaf, and V. Miši, "Performance of a beacon enabled IEEE 802.15.4 cluster with downlink and uplink traffic," IEEE Trans. Parallel Distrib. Syst., vol. 17, no. 4, pp. 361-376, Apr. 2006.

[4] S. Pollin, M. Ergen, S. C. Ergen, B. Bougard, L. Van der Perre, I. Moerman, A. Bahai, P. Varaiya, and F. Catthoor, "Performance analysis of slotted carrier sense IEEE 802.15.4 medium access layer," IEEE Trans. Wireless Commun., vol. 7, no. 9, pp. 3359-3371, Sep. 2008.

[5] P. Park, P. Di Marco, P. Soldati, C. Fischione, and K. H. Johansson, "A generalized Markov chain model for effective analysis of slotted IEEE 802.15.4," in Proc. IEEE MASS, 2009, pp. 130-139.

[6] C. Buratti and R. Verdone, "Performance analysis of IEEE 802.15.4 non beacon-enabled mode," IEEE Trans. Veh. Technol., vol. 58, no. 7, pp. 3480-3493, Sep. 2009.
[7] C. Y. Jung, H. Y. Hwang, D. K. Sung, and G. U. Hwang, "Enhanced Markov chain model and throughput analysis of the slotted CSMA/CA for IEEE 802.15.4 under unsaturated traffic conditions," IEEE Trans. Veh. Technol., vol. 58, no. 1, pp. 473-478, Jan. 2009.

[8] J. He, Z. Tang, H.-H. Chen, and Q. Zhang, "An accurate and scalable analytical model for IEEE 802.15.4 slotted CSMA/CA networks," IEEE Trans. Wireless Commun., vol. 8, no. 1, pp. 440-448, Jan. 2009.

[9] P. Park, P. Di Marco, C. Fischione, and K. H. Johansson, Modeling and optimization of the IEEE 802.15.4 protocol for reliable and timely communications, IEEE Trans. Parallel Distrib. Syst., to be published. [Online]. Available: http://doi.ieeecomputersociety.org/10.1109/TPDS.2012.159

[10] G. Bianchi, "Performance analysis of the IEEE 802.11 distributed coordination function," IEEE J. Sel. Areas Commun., vol. 18, no. 3, pp. 535-547, Mar. 2000

[11] J. Miši, S. Shafi, and V. Miši, "Maintaining reliability through activity management in an 802.15.4 sensor cluster," IEEE Trans. Veh. Technol., vol. 55, no. 3, pp. 779-788, May 2006.

[12] A. Faridi, M. R. Palattella, M. Dohler, G. Boggia, A. Grieco, P. Camarda, and A. Lozano, "Comprehensive evaluation of the IEEE 802.15.4 MAC layer performance with retransmissions," IEEE Trans. Veh. Technol., vol. 59, no. 8, pp. 3917-3932, Oct. 2010.

[13] D. Malone, K. Duffy, and D. Leith, "Modeling the 802.11 distributed coordination function in nonsaturated heterogeneous conditions," IEEE/ACM Trans. Netw., vol. 15, no. 1, pp. 159-172, Feb. 2007.

[14] M. Chen, G. Liu, D. Wu, and G. Zhu, "A unified model for performance analysis of 802.11 in heterogeneous traffic and saturation condition," in Proc. IEEE ICC, 2008, pp. 2644-2649.

[15] E. Ndih, N. Khaled, and G. De Micheli, "An analytical model for the contention access period of the slotted IEEE 802.15.4 with service differentiation," in Proc. IEEE ICC, 2009, pp. 1-6.

[16] T. Kim and J. Lim, "Throughput analysis considering coupling effect in IEEE 802.11 networks with hidden stations," IEEE Commun. Lett., vol. 13, no. 3, pp. 175-177, Mar. 2009.

[17] H. Wu, F. Zhu, Q. Zhang, and Z. Niu, "Analysis of IEEE 802.11 DCF with hidden terminals," in Proc. IEEE GLOBECOM, 2006, pp. 1-5.

[18] M. Hira, F. Tobagi, and K. Medepalli, "Throughput analysis of a path in an IEEE 802.11 multi-hop wireless network," in Proc. IEEE WCNC, 2007, pp. 441-446.

[19] J. Baras, V. Tabatabaee, P. Papageorgiou, and N. Rentz, "Modelling and optimization for multi-hop wireless networks using fixed point and automatic differentiation," in Proc. WiOPT, 2008, pp. 295-300.

[20] M. M. Carvalho and J. J. Garcia-Luna-Aceves, "A scalable model for channel access protocols in multi-hop ad hoc networks," in Proc. ACM MobiCom, New York, 2004, pp. 330-344.

[21] M. Martalo, S. Busanelli, and G. Ferrari, "Markov chain-based performance analysis of multi-hop IEEE 802.15.4 wireless networks," Perform. Eval., vol. 66, no. 12, pp. 722-741, Dec. 2009.

[22] S. C. Ergen, P. Di Marco, and C. Fischione, "MAC protocol engine for sensor networks," in Proc. IEEE GLOBECOM, 2009, pp. 1-8.

[23] D. P. Bertsekas and J. N. Tsitsiklis, Parallel and Distributed Computation: Numerical Methods. Belmont, MA: Athena Scientific, 1997.

[24] H. Takagi, Queueing Analysis: Finite Systems. Amsterdam, The Netherlands, 1993.

[25] A. R. Conn, N. Gould, and P. L. Toint, Trust-Region Methods. Philadelphia, PA: SIAM, 2000.

[26] P. Park, P. Di Marco, C. Fischione, and K. H. Johansson, "Accurate delay analysis of slotted IEEE 802.15.4 for control applications," Roy. Inst. Technol., Stockholm, Sweden, 2010, Tech. Rep. [Online]. Available: http://www.ee.kth.se/pgpark/papers/delay_wpan.pdf

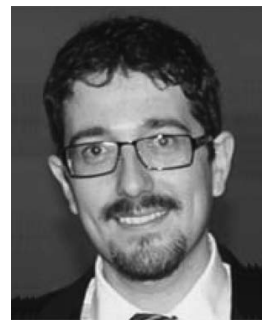

Piergiuseppe Di Marco (S'12) received the M.Sc. degree in telecommunications engineering from the University of L'Aquila, L'Aquila, Italy, in 2008. He is currently working toward the Ph.D. degree in telecommunications with the Automatic Control Laboratory, School of Electrical Engineering, Royal Institute of Technology, Stockholm, Sweden.

His research interests include modeling, design, and optimization for wireless sensor networks. 


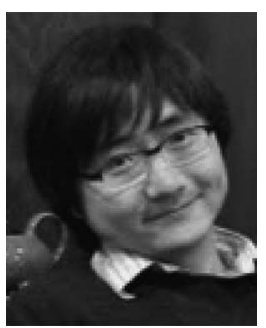

Pangun Park (M'12) received the M.S. and Ph.D. degrees in electrical engineering from the Royal Institute of Technology, Stockholm, Sweden, in 2007 and 2011, respectively.

Since 2011, he has been a Postdoctoral Researcher in electrical engineering and computer science with the University of California, Berkeley. His research interests include cyber-physical systems, wireless sensor and actuator networks, and networked control systems.

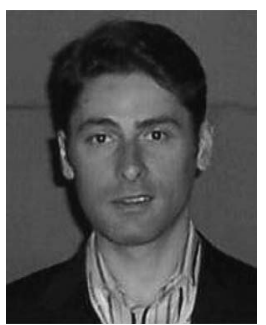

Carlo Fischione (M'06) received Dr.Eng. degree in electronic engineering and the Ph.D. degree in electrical and information engineering from the University of L'Aquila, L'Aquila, Italy, in 2001 and 2005, respectively.

$\mathrm{He}$ is a tenured Associate Professor with the School of Electrical Engineering and ACCESS Linnaeus Center, Royal Institute of Technology, Stockholm, Sweden. He held research positions with the University of California, Berkeley, and the Royal Institute of Technology. His research interests include optimization and parallel computation with applications to wireless sensor networks, networked control systems, and wireless networks.

Dr. Fischione is a Member of the Society of Industrial and Applied Mathematics. He has chaired or served as a technical member of program committees for several international conferences and is serving as referee for several technical journals. He received the Best Paper Award from the IEEE TRANSACTIONS ON INDUSTRIAL INFORMATICS and the Junior Research Award from the Swedish Research Council.

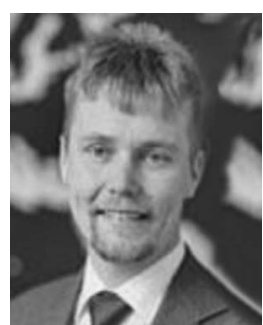

Karl Henrik Johansson (SM'08)received the M.Sc. and $\mathrm{Ph} . \mathrm{D}$. degrees in electrical engineering from Lund University, Lund, Sweden.

$\mathrm{He}$ is the Director of the ACCESS Linnaeus Center and a Professor with the School of Electrical Engineering, Royal Institute of Technology, Stockholm, Sweden. He is a Wallenberg Scholar and has held a six-year Senior Researcher Position with the Swedish Research Council. He has held visiting positions with the University of California, Berkeley, and the California Institute of Technology, Pasadena. He has been or is currently on the Editorial Board of the International Federation of Automatic Control (IFAC) journal Automatica, the IET Control Theory and Applications, and the International Journal of Robust and Nonlinear Control. His research interests are networked control systems, hybrid and embedded control, and control applications in automation, communication, and transportation systems.

Dr. Johansson was a member of the IEEE Control Systems Society Board of Governors and the Chair of the IFAC Technical Committee on Networked Systems. He has been on the Editorial Board of the IEEE TRANSACTIONS ON Automatic CONTROL. 